\title{
Relative set theory: Strong stability
}

\author{
KAREL HRBACEK
}

\begin{abstract}
GRIST is an axiomatic framework for nonstandard set theory with many "levels of standardness." The paper establishes a number of general consequences of GRIST, in particular, a very strong form of Transfer principle.

2010 Mathematics Subject Classification 26E35 (primary); 03E70, 03H05 (secondary)
\end{abstract}

Keywords: nonstandard analysis, nonstandard set theory, IST, RIST, level, relative infinitesimal, transfer, S-continuity

This is the last in a series of three articles devoted to GRIST, an axiomatic presentation of nonstandard analysis with many "levels of standardness." The two previous papers, [4] and [5], will be referred to as RST and RST2, respectively.

It is shown in RST that GRIST is complete over ZFC: If an extension of GRIST proves a theorem that is not provable in GRIST, then it proves a theorem in the language of ZFC that is not provable in $\mathbf{Z F C}$ [see Proposition 6.5]. In other words, no additional principles can be added to GRIST while keeping it conservative over ZFC. Yet in mathematical applications it is sometimes awkward to argue directly from the axioms of GRIST. It is convenient to have at one's disposal other principles, provable in GRIST, but tailor-made for certain kinds of applications. A number of such consequences of GRIST is derived in RST, Section 12; see also RST2, Proposition 1.10. For applications of relative set theory see RST2 and $[3,6,10]$.

This paper focuses on deducing some further useful principles in GRIST. Foremost among them is Strong Stability, perhaps the ultimate generalization of Transfer. Section 1 begins with a formulation of Strong Stability. Strong Stability is then used to prove that levels represented by elements of a given set are precisely those from a finite union of singletons and closed intervals (in the ordering of levels by inclusion).

Section 2 contains the proof of Strong Stability in GRIST. It relies heavily on the development of GRIST in RST. Counterexamples to some "natural" strengthenings of Strong Stability are also constructed there.

Section 3 deals with some variants of Idealization and Choice that are provable in GRIST. It also presents a generalization of Robinson's Lemma due to Andreev. 
In Section 4 we complete the study of $\left(\mathbf{V}_{1}, \mathbf{V}_{2}\right)$-continuity initiated in RST2.

In GRIST, the ordering of levels is dense (and there is a coarsest level). Alternatively, one can postulate that levels are discretely ordered (each level has an immediate successor, and each but the coarsest level has an immediate predecessor). There are other possibilities, and weaker theories (FRIST [2], Péraire's RIST [9]) agnostic on the details of the ordering of levels. In Section 5 we show that all these theories prove exactly the same open formulas (formulas where no quantification over levels occurs).

Finally, in Section 6 we fill a gap in the proof of Proposition 6.10 in RST, and add a few easy but useful observations on GRIST that have not been made explicit in RST.

For convenient reference, the axioms of GRIST are listed below [see RST2, page 4]. In addition to $\mathbf{Z F C}$, they are

Relativization The conjunction of:

(o) $(\forall \mathbf{U}, \mathbf{V})[(\forall x)(x \in \mathbf{U} \leftrightarrow x \in \mathbf{V}) \rightarrow \mathbf{U}=\mathbf{V}]$;

(i) $(\forall x)(\exists \mathbf{V})[x \in \mathbf{V} \wedge(\forall \mathbf{U})(x \in \mathbf{U} \rightarrow \mathbf{V} \subseteq \mathbf{U})]$

(this uniquely determined level $\mathbf{V}$ is denoted $\mathbf{V}(x) ; \mathbf{V}(\cdot):=\mathbf{V}(0)$ is the coarsest level);

(ii) $(\forall \mathbf{V})[0 \in \mathbf{V} \wedge(\exists x \in \mathbf{V})(\forall \mathbf{U})(x \in \mathbf{U} \rightarrow \mathbf{V} \subseteq \mathbf{U})]$;

(iii) $(\forall \mathbf{U}, \mathbf{V})(\mathbf{U} \subseteq \mathbf{V} \vee \mathbf{V} \subseteq \mathbf{U})$;

(iv) $(\forall \mathbf{U})(\exists \mathbf{V})(\mathbf{U} \subset \mathbf{V})$;

(v) $(\forall \mathbf{U}, \mathbf{V})(\mathbf{U} \subset \mathbf{V} \rightarrow(\exists \mathbf{W})(\mathbf{U} \subset \mathbf{W} \subset \mathbf{V})$.

\section{Transfer (or Stability)}

For all $\mathbf{U} \subseteq \mathbf{V}$ and all $x_{1}, \ldots, x_{k} \in \mathbf{U}, \quad \mathcal{P}\left(x_{1}, \ldots, x_{k} ; \mathbf{U}\right) \leftrightarrow \mathcal{P}\left(x_{1}, \ldots, x_{k} ; \mathbf{V}\right)$.

\section{Standardization}

For all $\mathbf{U}$ and all $A, x_{1}, \ldots, x_{k}$, either $(\forall \mathbf{V})(\mathbf{U} \subseteq \mathbf{V})$ or there exist $\mathbf{V} \subset \mathbf{U}$ and $B \in \mathbf{V}$ such that, for every $\mathbf{W}$ with $\mathbf{V} \subseteq \mathbf{W} \subset \mathbf{U}$,

$$
(\forall y \in \mathbf{W})\left(y \in B \leftrightarrow y \in A \wedge \mathcal{P}\left(y, x_{1}, \ldots, x_{k} ; \mathbf{W}\right)\right) .
$$

\section{Idealization}

For all $\mathbf{U}, \mathbf{V}, A$ such that $A \in \mathbf{U} \subset \mathbf{V}$, and all $x_{1}, \ldots, x_{k}$,

$\left(\forall a \in \mathcal{P}^{\text {fin }} A\right)(\forall \mathbf{W} \subset \mathbf{V})\left[a \in \mathbf{W} \rightarrow(\exists y)(\forall x \in a) \mathcal{P}\left(x, y, x_{1}, \ldots, x_{k} ; \mathbf{V}\right)\right] \leftrightarrow$

$(\exists y)(\forall x \in A)(\forall \mathbf{W} \subset \mathbf{V})\left[x \in \mathbf{W} \rightarrow \mathcal{P}\left(x, y, x_{1}, \ldots, x_{k} ; \mathbf{V}\right)\right]$

\section{Granularity}

For all $x_{1}, \ldots, x_{k}$, if $(\exists \mathbf{U}) \mathcal{P}\left(x_{1}, \ldots, x_{k} ; \mathbf{U}\right)$, then

$$
(\exists \mathbf{U})\left[\mathcal{P}\left(x_{1}, \ldots, x_{k} ; \mathbf{U}\right) \wedge(\forall \mathbf{V})\left(\mathbf{V} \subset \mathbf{U} \rightarrow \neg \mathcal{P}\left(x_{1}, \ldots, x_{k} ; \mathbf{V}\right)\right)\right]
$$




\section{Strong Stability and 'sets of levels'.}

The key principle of GRIST is Transfer, also referred to as Stability:

For all $\mathbf{V} \subseteq \mathbf{V}^{\prime}$ and all $x_{1}, \ldots, x_{k} \in \mathbf{V}$,

$$
\mathcal{P}\left(x_{1}, \ldots, x_{k} ; \mathbf{V}\right) \leftrightarrow \mathcal{P}\left(x_{1}, \ldots, x_{k} ; \mathbf{V}^{\prime}\right),
$$

where $\mathcal{P}\left(x_{1}, \ldots, x_{k} ; \mathbf{V}\right)$ is any $\mathbf{V}$-formula, ie, a formula where all quantifiers over levels are of the form $(\forall \mathbf{W} \supseteq \mathbf{V})$ or $(\exists \mathbf{W} \supseteq \mathbf{V})$.

The most irksome limitation of Transfer is the restriction $x_{1}, \ldots, x_{k} \in \mathbf{V}$; but without it the principle fails as stated [consider $\mathbf{V} \subset \mathbf{V}^{\prime}, x \in \mathbf{V}^{\prime} \backslash \mathbf{V}$, and the formula $\mathcal{P}(x ; \mathbf{V})$ : $x \in \mathbf{V}$ ]. The first step towards transgressing this limitation is made in the Local Transfer principle.

Local Transfer [RST2, Proposition 1.10 (6)]: For any sets $x_{k+1}, \ldots, x_{n}$ and any $\mathbf{V}_{0}$ there is $\mathbf{V}^{\prime} \supset \mathbf{V}_{0}$ such that, for all $\mathbf{V}_{0} \subseteq \mathbf{V} \subset \mathbf{V}^{\prime}$ and all $x_{1}, \ldots, x_{k} \in \mathbf{V}_{0}$,

$$
\mathcal{P}\left(x_{1}, \ldots, x_{k}, x_{k+1}, \ldots, x_{n} ; \mathbf{V}_{0}\right) \leftrightarrow \mathcal{P}\left(x_{1}, \ldots, x_{k}, x_{k+1}, \ldots, x_{n} ; \mathbf{V}\right)
$$

We also have

Support Principle [RST2, Proposition 1.10 (5)]: Given a $\mathbf{V}$-formula $\mathcal{P}\left(x_{1}, \ldots, x_{k} ; \mathbf{V}\right)$ and sets $x_{1}, \ldots, x_{k}$, there is a finite set $\left\{v_{0}, v_{1}, \ldots, v_{n}\right\}$ such that $\mathbf{V}(\cdot)=\mathbf{V}\left(v_{0}\right) \subset$ $\mathbf{V}\left(v_{1}\right) \subset \ldots \subset \mathbf{V}\left(v_{n}\right)$ and for all $i \leq n$ and all $\mathbf{V}$ with $\mathbf{V}\left(v_{i}\right) \subseteq \mathbf{V} \subset \mathbf{V}\left(v_{i+1}\right)$ $\left[\mathbf{V}\left(v_{i}\right) \subseteq \mathbf{V}\right.$ if $\left.i=n\right]$,

$$
\mathcal{P}\left(x_{1}, \ldots, x_{k} ; \mathbf{V}\left(v_{i}\right)\right) \leftrightarrow \mathcal{P}\left(x_{1}, \ldots, x_{k} ; \mathbf{V}\right) \leftrightarrow \neg \mathcal{P}\left(x_{1}, \ldots, x_{k} ; \mathbf{V}\left(v_{i+1}\right)\right) .
$$

In this section we formulate a principle (Strong Stability) that generalizes all of the above, and give an example that illustrates its use.

Definition 1.1 (RST, Definition 8.3; see also RST, Proposition 8.6, and Section 2 of this paper.) A set $L$ is a level set if for all $x, y \in L, \mathbf{V}(x)=\mathbf{V}(y)$ implies $x=y$.

Level sets are finite, and the relation $\sqsubseteq$ defined on $L$ by $x \sqsubseteq y \leftrightarrow \mathbf{V}(x) \subseteq \mathbf{V}(y)$ is a well-ordering. We always describe level sets in the increasing order by $\sqsubseteq$; ie, if $L=\left\{z_{0}, z_{1}, \ldots, z_{\ell}\right\}$ is a level set, then $\mathbf{V}\left(z_{0}\right) \subset \mathbf{V}\left(z_{1}\right) \subset \ldots \subset \mathbf{V}\left(z_{\ell}\right)$.

Definition 1.2 Let $L$ be a level set. We write $\mathbf{V} \cong_{L} \mathbf{V}^{\prime}$ if $\mathbf{V} \subseteq \mathbf{V}(z) \leftrightarrow \mathbf{V}^{\prime} \subseteq \mathbf{V}(z)$ and $\mathbf{V}(z) \subseteq \mathbf{V} \leftrightarrow \mathbf{V}(z) \subseteq \mathbf{V}^{\prime}$ hold for all $z \in L$.

In other words, if $L=\left\{z_{0}, z_{1}, \ldots, z_{\ell}\right\}$, then $\mathbf{V} \cong_{L} \mathbf{V}^{\prime}$ means that either $\mathbf{V}=\mathbf{V}^{\prime}=\mathbf{V}\left(z_{j}\right)$ for some $j \leq \ell$, or $\mathbf{V}, \mathbf{V}^{\prime} \subset \mathbf{V}\left(z_{0}\right)$, or $\mathbf{V}\left(z_{j}\right) \subset \mathbf{V}, \mathbf{V}^{\prime} \subset \mathbf{V}\left(z_{j+1}\right)$ for some $j<\ell$, or $\mathbf{V}\left(z_{\ell}\right) \subset \mathbf{V}, \mathbf{V}^{\prime}$. Thus $\cong_{L}$ classifies all levels into $2 \ell+3$ classes. 
Recall [RST2, page 4] that $\mathcal{P}\left(x_{1}, \ldots, x_{k}, y_{1}, \ldots, y_{\ell} ; \mathbf{V}, \mathbf{V}_{1}, \ldots, \mathbf{V}_{n}\right)$ denotes a formula of the language of GRIST where all quantifiers over levels are of the form $(\forall \mathbf{W} \supseteq \mathbf{V})$ or $(\exists \mathbf{W} \supseteq \mathbf{V})$. For brevity, we often write $\bar{x}$ and $\bar{y}$ for the sequences $x_{1}, \ldots, x_{k}$ and $y_{1}, \ldots, y_{\ell}$, respectively. Then $\langle\bar{x}\rangle$ denotes $\left\langle x_{1}, \ldots, x_{k}\right\rangle$, etc.

STRONG STABILITY: Given $y_{1}, \ldots, y_{\ell}$, there is a level set $L$ such that if $\mathbf{V} \subset \mathbf{V}_{1}$, $\mathbf{V} \subset \mathbf{V}_{1}^{\prime}$ and $\mathbf{V}_{1} \cong_{L} \mathbf{V}_{1}^{\prime}$, then

$$
(\forall \bar{x} \in \mathbf{V})\left(\mathcal{P}\left(\bar{x}, \bar{y} ; \mathbf{V}, \mathbf{V}_{1}\right) \leftrightarrow \mathcal{P}\left(\bar{x}, \bar{y} ; \mathbf{V}, \mathbf{V}_{1}^{\prime}\right)\right)
$$

The proof is postponed until Section 2, where also a stronger version, for several levels simultaneously, can be found. Here we give a simple example of an application of this principle. We show that every "set of levels" is a finite union of singletons and closed intervals in the linear ordering of levels by $\subseteq$.

Theorem 1.3 For every $X \neq \varnothing$ there exist level sets $L=\left\{\alpha_{0}, \ldots, \alpha_{n}\right\}$ and $L^{\prime}=$ $\left\{\alpha_{0}^{\prime}, \ldots, \alpha_{n}^{\prime}\right\}$ such that $\mathbf{V}\left(\alpha_{i}\right) \subseteq \mathbf{V}\left(\alpha_{i}^{\prime}\right)$ for all $i \leq n, \mathbf{V}\left(\alpha_{i}^{\prime}\right) \subset \mathbf{V}\left(\alpha_{i+1}\right)$ for all $i<n$, and

$$
\begin{aligned}
& (\forall x \in X)(\exists i \leq n)\left(\mathbf{V}\left(\alpha_{i}\right) \subseteq \mathbf{V}(x) \subseteq \mathbf{V}\left(\alpha_{i}^{\prime}\right)\right), \\
& (\forall \mathbf{V})(\forall i \leq n)\left[\mathbf{V}\left(\alpha_{i}\right) \subseteq \mathbf{V} \subseteq \mathbf{V}\left(\alpha_{i}^{\prime}\right) \rightarrow(\exists x \in X)(\mathbf{V}(x)=\mathbf{V})\right]
\end{aligned}
$$

if $X$ is finite. If $X$ is infinite, (1) and (2) hold with $\mathbf{V}\left(\alpha_{n}\right) \subseteq \mathbf{V}(x) \subseteq \mathbf{V}\left(\alpha_{n}^{\prime}\right)$ and $\mathbf{V}\left(\alpha_{n}\right) \subseteq \mathbf{V} \subseteq \mathbf{V}\left(\alpha_{n}^{\prime}\right)$ replaced by $\mathbf{V}\left(\alpha_{n}\right) \subseteq \mathbf{V}(x)$ and $\mathbf{V}\left(\alpha_{n}\right) \subseteq \mathbf{V}$, respectively.

Lemma 1.4 If $\mathbf{V}(\alpha) \subset \mathbf{V}\left(\alpha^{\prime}\right)$ and $(\exists x \in X)(\mathbf{V}(x)=\mathbf{V})$ holds for all $\mathbf{V}$ such that $\mathbf{V}(\alpha) \subset \mathbf{V} \subset \mathbf{V}\left(\alpha^{\prime}\right)$, then also

$$
\begin{aligned}
& (\exists x \in X)(\mathbf{V}(x)=\mathbf{V}(\alpha)) \text { and } \\
& (\exists x \in X)\left(\mathbf{V}(x)=\mathbf{V}\left(\alpha^{\prime}\right)\right)
\end{aligned}
$$

Proof (B) Let $a$ be a finite set, $\mathbf{V}(a) \subset \mathbf{V}\left(\alpha^{\prime}\right)$. Fix $\mathbf{V}$ such that $\mathbf{V}(a), \mathbf{V}(\alpha) \subset \mathbf{V} \subset$ $\mathbf{V}\left(\alpha^{\prime}\right)$ and $x \in X$ such that $\mathbf{V}(x)=\mathbf{V}$. By RST2, Proposition 1.10 (13), $y \in a$ implies $y \in \mathbf{V}(a)$, so $y \neq x$. Hence $\left(\forall^{\text {fin }} a\right)\left[\mathbf{V}(a) \subset \mathbf{V}\left(\alpha^{\prime}\right) \rightarrow\left(\exists x \in \mathbf{V}\left(\alpha^{\prime}\right)\right)(\forall y \in a)(x \in\right.$ $X \wedge y \neq x)$ ]. By GRIST Idealization we obtain $x \in X, x \in \mathbf{V}\left(\alpha^{\prime}\right)$, such that $y \neq x$ holds for all $y \in X$ with $\mathbf{V}(y) \subset \mathbf{V}\left(\alpha^{\prime}\right)$. Then $x \in X$ and $\mathbf{V}(x)=\mathbf{V}\left(\alpha^{\prime}\right)$.

(A) If $\mathbf{V}(\alpha)=\mathbf{V}(\cdot)$ [the coarsest level], consider the statement

$$
\mathcal{P}(X ; \mathbf{V}): \quad(\exists x \in X)(x \in \mathbf{V})
$$


$\mathcal{P}(X ; \mathbf{V})$ holds for all $\mathbf{V}$ such that $\mathbf{V}(\cdot) \subset \mathbf{V} \subset \mathbf{V}\left(\alpha^{\prime}\right)$, by the assumption. By Granularity, there is a coarsest level $\mathbf{V}$ for which $\mathcal{P}(X ; \mathbf{V})$ holds. From density of levels [Relativization (v)] we conclude that $\mathcal{P}(X ; \mathbf{V}(\cdot)):(\exists x \in X)(x \in \mathbf{V}(\cdot))$ holds.

If $\mathbf{V}(\cdot) \subset \mathbf{V}(\alpha)$ and for every $\mathbf{V} \subset \mathbf{V}(\alpha)$ there is $x \in X$ such that $\mathbf{V} \subseteq \mathbf{V}(x) \subset \mathbf{V}(\alpha)$, the argument in the proof of (B) [with $\mathbf{V}(\cdot)$ in place of $\mathbf{V}(\alpha)$ and $\mathbf{V}(\alpha)$ in place of $\mathbf{V}\left(\alpha^{\prime}\right)$ ] shows that $(\exists x \in X)(\mathbf{V}(x)=\mathbf{V}(\alpha))$.

It remains to consider the case when there is a $\mathbf{V} \subset \mathbf{V}(\alpha)$ such that $\neg(\exists x \in X)(\mathbf{V} \subseteq$ $\mathbf{V}(x) \subset \mathbf{V}(\alpha))$. Fix such $\mathbf{V}=: \overline{\mathbf{V}}$. For every finite $a$ with $\mathbf{V}(a) \subset \overline{\mathbf{V}}$ there is $Y \in \overline{\mathbf{V}}, Y$ finite, such that $(\forall x \in a)(x \in X \rightarrow x \in Y)$ [let $Y:=a$ ]. By GRIST Idealization, there is a finite $Y \in \overline{\mathbf{V}}$ such that $(\forall x \in X)(\mathbf{V}(x) \subset \overline{\mathbf{V}} \rightarrow x \in Y)$. Let $Z:=X \backslash Y$ and note that $(\forall x)(x \in Z \leftrightarrow x \in X \wedge \mathbf{V}(\alpha) \subseteq \mathbf{V}(x))[Y \in \overline{\mathbf{V}}$ is finite, so $x \in Y \rightarrow x \in \overline{\mathbf{V}}$, by RST2, Proposition 1.10 (13)]. As in the proof of the $\mathbf{V}(\alpha)=\mathbf{V}(\cdot)$ case, we consider

$$
\mathcal{P}(Z ; \mathbf{V}): \quad(\exists x \in Z)(x \in \mathbf{V})
$$

We observe that $\mathcal{P}(Z ; \mathbf{V}) \leftrightarrow \mathcal{P}(X ; \mathbf{V})$ for $\mathbf{V} \supseteq \mathbf{V}(\alpha)$, and hence $\mathcal{P}(Z ; \mathbf{V})$ holds for all $\mathbf{V}$ such that $\mathbf{V}(\alpha) \subset \mathbf{V} \subset \mathbf{V}\left(\alpha^{\prime}\right)$. Also, $\mathcal{P}(Z ; \mathbf{V})$ fails for $\mathbf{V} \subset \mathbf{V}(\alpha)$. By Granularity, $\mathcal{P}(Z ; \mathbf{V}(\alpha))$ holds. Hence $(\exists x \in Z)(x \in \mathbf{V}(\alpha))$; as $x \in Z \rightarrow \mathbf{V}(x) \supseteq \mathbf{V}(\alpha)$, we have $x \in Z \subseteq X$ and $\mathbf{V}(x)=\mathbf{V}(\alpha)$.

Proof of Theorem 1.3 We apply Strong Stability to the statement

$$
\mathcal{P}(X ; \mathbf{V}(\cdot), \mathbf{V}): \quad(\exists x \in X)(\mathbf{V}(x)=\mathbf{V})
$$

[in detail: $(\exists x \in X)(\forall \mathbf{W} \supseteq \mathbf{V}(\cdot))(x \in \mathbf{W} \leftrightarrow \mathbf{V} \subseteq \mathbf{W})$ ] and obtain a level set $M=$ $\left\{\gamma_{0}, \ldots, \gamma_{k}\right\}$ such that, wlog, $\mathbf{V}\left(\gamma_{0}\right)=\mathbf{V}(\cdot)$ and for all $i \leq k, \mathbf{V}\left(\gamma_{i}\right) \subset \mathbf{V}, \mathbf{V}^{\prime} \subset \mathbf{V}\left(\gamma_{i+1}\right)$ $\left[\mathbf{V}\left(\gamma_{i}\right) \subset \mathbf{V}, \mathbf{V}^{\prime}\right.$ if $\left.i=k\right]$ implies $\mathcal{P}(X ; \mathbf{V}(\cdot), \mathbf{V}) \leftrightarrow \mathcal{P}\left(X ; \mathbf{V}(\cdot), \mathbf{V}^{\prime}\right)$. By Lemma 1.4, if $\mathcal{P}(X ; \mathbf{V}(\cdot), \mathbf{V})$ holds for all $\mathbf{V}\left(\gamma_{i}\right) \subset \mathbf{V} \subset \mathbf{V}\left(\gamma_{i+1}\right)$, then also $\mathcal{P}\left(X ; \mathbf{V}(\cdot), \mathbf{V}\left(\gamma_{i}\right)\right)$ and $\mathcal{P}\left(X ; \mathbf{V}(\cdot), \mathbf{V}\left(\gamma_{i+1}\right)\right)$ hold. By Proposition 6.4 proved in Section 6 and Examples (2) and (3) that precede it, the sets $\{z \in M:(\exists x \in X)(\mathbf{V}(x)=\mathbf{V}(z))\}$ and $\{z \in M$ : $(\forall \mathbf{V})[\mathbf{V} \supset \mathbf{V}(z) \rightarrow(\exists x \in X)(\mathbf{V}(z) \subset \mathbf{V}(x) \subseteq \mathbf{V})]\}$ exist. From these sets one easily obtains the sets $L$ and $L^{\prime}$ as in the Theorem, by amalgamating adjacent intervals when necessary.

By RST2, Proposition 2.15, the level sets $L$ and $L^{\prime}$ can be taken to be sets of natural numbers: $L=\left\{k_{0}, \ldots, k_{n}\right\}$ and $L^{\prime}=\left\{k_{0}^{\prime}, \ldots, k_{n}^{\prime}\right\}$, with $\mathbf{V}\left(k_{i}\right) \subseteq \mathbf{V}\left(k_{i}^{\prime}\right)$ for all $i \leq n$ and $\mathbf{V}\left(k_{i}^{\prime}\right) \subset \mathbf{V}\left(k_{i+1}\right)$ for all $i<n$. We can assume that $\mathbf{V}\left(k_{i}\right)=\mathbf{V}\left(k_{i}^{\prime}\right)$ implies $k_{i}=k_{i}^{\prime} .\left[\left\{k_{i}: \mathbf{V}\left(k_{i}\right)=\mathbf{V}\left(k_{i}^{\prime}\right)\right\}\right.$ is a set, again by Proposition 6.4, because the formula $(\exists i \leq n)\left(z=k_{i} \wedge z \sqsubseteq k_{i}^{\prime} \wedge k_{i}^{\prime} \sqsubseteq z\right)$ is stable in $z$.] 
Let $\left[k, k^{\prime}\right]:=\left\{i \in \mathbb{N}: k \leq i \leq k^{\prime}\right\}$ and $[k, \infty):=\{i \in \mathbb{N}: k \leq i\}$. We define $S^{\prime}:=\bigcup_{i<n}\left[k_{i}, k_{i}^{\prime}\right]$ and $S:=S^{\prime} \cup\left[k_{n}, k_{n}^{\prime}\right]$ if $X$ is finite; $S:=S^{\prime} \cup\left[k_{n}, \infty\right)$ if $X$ is infinite. Then

$$
(\forall x \in X)(\exists i \in S)(\mathbf{V}(x)=\mathbf{V}(i)) \text { and }(\forall i \in S)(\exists x \in X)(\mathbf{V}(x)=\mathbf{V}(i))
$$

In the terminology introduced on page 12, every set is level-equivalent to a finite union of singletons and closed intervals on $\mathbb{N}$.

\section{Proof of Strong Stability in GRIST.}

This section relies heavily on the development of GRIST as given in RST. For this reason, it is convenient here to work with the original formulation of GRIST in terms of $\sqsubseteq$, rather than use the language of levels $\left(\right.$ GRIST $\left.^{\circledR}\right)$ introduced in RST2.

In the $\in-\sqsubseteq$-language, $L$ is a level set if for all $x, y \in L, x \boxminus y$ implies $x=y$. We recall that $x \boxminus y$ is shorthand for $x \sqsubseteq y \wedge y \sqsubseteq x$.

We write $\alpha \cong_{L} \beta$ if $\alpha \sqsubseteq \gamma \leftrightarrow \beta \sqsubseteq \gamma$ and $\gamma \sqsubseteq \alpha \leftrightarrow \gamma \sqsubseteq \beta$ hold for all $\gamma \in L$.

In other words, if $L=\left\{\gamma_{0}, \gamma_{1}, \ldots, \gamma_{\ell}\right\}$, then $\alpha \cong_{L} \beta$ means that either $\alpha \boxminus \beta \boxminus \gamma_{j}$ for some $j \leq \ell$, or $\alpha, \beta \sqsubset \gamma_{0}$, or $\gamma_{j} \sqsubset \alpha, \beta \sqsubset \gamma_{j+1}$ for some $j<\ell$, or $\gamma_{\ell} \sqsubset \alpha, \beta$.

$\mathcal{P}\left(x_{1}, \ldots, x_{k}, y_{1}, \ldots, y_{\ell} ; z_{1}, \ldots, z_{n}\right)$ denotes a formula of the $\in$ - $\sqsubseteq$-language where the variables $z_{1}, \ldots, z_{n}$ appear only in the scope of $\sqsubseteq$ [RST, Definition 12.25].

The Strong Stability principle in this language goes as follows [we recall that $\mathbb{S}_{\alpha}:=$ $\{x: x \sqsubseteq \alpha\}$, and $\mathcal{P}^{\alpha}$ is the formula obtained from $\mathcal{P}$ by replacing every occurrence of $\sqsubseteq$ with $\sqsubseteq_{\alpha}$, defined by $\left.x \sqsubseteq \alpha y \leftrightarrow x \sqsubseteq y \vee x \sqsubseteq \alpha\right]$.

Theorem 2.1 (Strong Stability) Given $y_{1}, \ldots, y_{\ell}$, there is a level set $L$, independent of $\mathcal{P}$, such that if $\alpha \sqsubset \beta, \alpha \sqsubset \beta^{\prime}$ and $\beta \cong_{L} \beta^{\prime}$, then

$$
\left(\forall \bar{x} \in \mathbb{S}_{\alpha}\right)\left(\mathcal{P}^{\alpha}(\bar{x}, \bar{y} ; \beta) \leftrightarrow \mathcal{P}^{\alpha}\left(\bar{x}, \bar{y} ; \beta^{\prime}\right)\right) .
$$

One can take $L=\operatorname{ran} \vec{u}$ where $\vec{u}$ is a pedigree for $\langle\bar{y}\rangle$ over some $A \in \mathbb{S}_{0}$. The notion of pedigree is the key technical tool for detailed study of GRIST. Roughly speaking, types of objects in the universe of GRIST are described by stratified ultrafilters. Pedigrees are finite sequences of stratified ultrafilters that describe, level by level, how the object realizes its type. We state the definitions of these concepts here for convenience [see RST, Sections 9 and 10]. 
We recall that $\boldsymbol{\beta} X$ is the set of all ultrafilters over $X$ (the Stone-Čech space over $X$ ), and $U \sim V$ means that $U \cap V$ is an ultrafilter. For an arbitrary nonempty set $A$ we define by recursion on ordinals:

(0) $\boldsymbol{\beta}_{0} A:=A$.

(1) For $\xi>0, \boldsymbol{\beta}_{<\xi} A:=\bigcup_{\eta<\xi} \boldsymbol{\beta}_{\eta} A$ and $\boldsymbol{\beta}_{\xi} A:=\boldsymbol{\beta}_{<\xi} A \cup\left\{U \in \boldsymbol{\beta}\left(\boldsymbol{\beta}_{<\xi} A\right): U\right.$ is nonprincipal and $\boldsymbol{\beta}_{<\eta} A \notin U$ for any $\left.\eta<\xi\right\}=\boldsymbol{\beta}_{<\xi} A \cup\left\{U \in \boldsymbol{\beta}\left(\boldsymbol{\beta}_{<\xi} A\right): U\right.$ is nonprincipal and there is no $V \in \boldsymbol{\beta}_{<\xi} A$ such that $\left.U \sim V\right\}$.

Elements of $\boldsymbol{\beta}_{\infty} A:=\bigcup_{\xi \in \mathbb{O} n} \boldsymbol{\beta}_{\xi} A$ are called stratified ultrafilters over $A$. For $U \in \boldsymbol{\beta}_{\infty} A$ we let $\operatorname{Dom} U:=A$. As usual, the recursive definition assigns to each stratified ultrafilter an ordinal rank. Stratified ultrafilters of rank 1 are the nonprincipal ultrafilters over $A$; stratified ultrafilters of rank 2 are the nonprincipal ultrafilters over $\boldsymbol{\beta} A$ that concentrate on nonprincipal ultrafilters over $A$ [ie, such that $(\boldsymbol{\beta} A \backslash A) \in U$ ], and so on.

Let $x \in A \in \mathbb{S}_{\alpha}$. An $\alpha$-pedigree for $x$ over $A$ is a sequence $\vec{u}=\left\langle u_{n}: n \leq \nu\right\rangle$ where $\nu \in \omega$ and

(i) every $u_{n}$ is a stratified ultrafilter over $A\left[\mathrm{ie}, u_{n} \in \boldsymbol{\beta}_{\infty} A\right]$;

(ii) $u_{0} \sqsubseteq \alpha ; u_{\nu}=x$;

(iii) $\alpha \sqsubset u_{1} \wedge(\forall n, m)\left(1 \leq n<m \leq \nu \rightarrow u_{n} \sqsubset u_{m}\right)$;

(iv) $\left(\forall z \sqsubset u_{n+1}\right)\left(z \in u_{n} \rightarrow u_{n+1} \in z\right)$, for all $n<\nu$.

The ultrafilter $u_{0}$ is called the $\alpha$-type of $x$ over $A$ and denoted $\operatorname{tp}_{\alpha}(x ; A)$. We also use $\vec{u}^{+}:=\left\langle u_{n}: 0<n \leq \nu\right\rangle$. We write $x \mathbf{M}_{\alpha} U$ as shorthand for: "There exists a [good; see Proposition 2.7] $\alpha$-pedigree $\vec{u}=\left\langle u_{n}: n \leq \nu\right\rangle$ for $x$ over some $A \in \mathbb{S}_{\alpha}$ such that $U=u_{0}$," and note that $U$ is then an $\alpha$-type of $x$. Pedigree and type mean 0 -pedigree and 0-type, respectively.

We also recall that, for $a \in A$ and $U \in \boldsymbol{\beta}_{\xi} B, U_{a} \in \boldsymbol{\beta}_{\xi}(A \times B)$ is the unique stratified ultrafilter such that $\overline{\pi_{1}}\left(U_{a}\right)=a$ and $\overline{\pi_{2}}\left(U_{a}\right)=U$ [RST, Proposition 9.7].

The main technical result needed for the proof of Theorem 2.1 is the following proposition.

Proposition 2.2 Let $\vec{u}=\left\langle u_{0}, u_{1}, \ldots, u_{i}, u_{i+1}, \ldots, u_{\nu}\right\rangle$ be a pedigree for $a \in A \in \mathbb{S}_{0}$ over $A$, and let $V, B \in \mathbb{S}_{0}, V \in \boldsymbol{\beta}_{1} B \backslash \boldsymbol{\beta}_{0} B$. Let $u_{i} \sqsubset \beta \sqsubset u_{i+1}\left[u_{\nu} \sqsubset \beta\right.$ if $\left.i=\nu\right]$, and let $b \boxminus \beta$ be such that $b \mathbf{M}_{0} V$ [ie, $b$ has the pedigree $\langle V, b\rangle$ over $B$; see $R S T$, Proposition 12.15]. Then the pedigree $\vec{v}$ for $\langle b, a\rangle$ over $B \times A$ has the form

$$
\left\langle v_{0}, \ldots, v_{m},\left(u_{i}\right)_{b},\left(u_{i+1}\right)_{b}, \ldots,\left(u_{\nu}\right)_{b}\right\rangle
$$

where $v_{m}=u_{i}^{\bowtie} V$ is defined in Proposition 2.4 below, and $v_{0}, \ldots, v_{m}$ are independent of the choice of $\beta$ and $b$. 
Proposition 2.2 shows how to extend a pedigree for a set $a$ to a pedigree that also "fixes" a particular level $\beta$. The proof requires two lemmas.

Lemma 2.3 $\left\langle\left(u_{i}\right)_{b},\left(u_{i+1}\right)_{b}, \ldots,\left(u_{\nu}\right)_{b}\right\rangle$ is the $\beta$-pedigree for $\langle b, a\rangle$ over $B \times A$.

Proof Let $f: A \rightarrow B \times A$ be defined by $f(x)=\langle b, x\rangle$. By RST, Theorem 10.10 [see also RST, Proposition 10.14], the range of the $\beta$-pedigree for $\langle b, a\rangle$ is $\left\{\bar{f}\left(u_{i}\right), \bar{f}\left(u_{i+1}\right), \ldots, \bar{f}\left(u_{\nu}\right)\right\}$. We prove that $\bar{\pi}_{1}\left(\bar{f}\left(u_{j}\right)\right)=b$ and $\overline{\pi_{2}}\left(\bar{f}\left(u_{j}\right)\right)=u_{j}$, for all $i \leq j \leq \nu$. We begin with noticing that $\pi_{1}(f(x))=b$ and $\pi_{2}(f(x))=x$. It is easily verified, from RST, Definition 9.3, that $\overline{k_{b}}(u)=b$ for all $u \in \boldsymbol{\beta}_{\infty} A$, where $k_{b}: A \rightarrow B$ is the constant function with value $b$. From this and RST, Proposition 9.4, we get $\overline{\pi_{1}}\left(\bar{f}\left(u_{j}\right)\right)=\overline{\pi_{1} \circ f}\left(u_{j}\right)=\overline{k_{b}}\left(u_{j}\right)=b$ and $\overline{\pi_{2}}\left(\bar{f}\left(u_{j}\right)\right)=\overline{\pi_{2} \circ f}\left(u_{j}\right)=\overline{I d_{A}}\left(u_{j}\right)=u_{j}$.

From the uniqueness in RST, Proposition 9.7, it follows that $\bar{f}\left(u_{j}\right)=\left(u_{j}\right)_{b}$. Finally, we notice that $\left(u_{j}\right)_{b} \boxminus_{\beta} u_{j}$ : we have $\left(u_{j}\right)_{b} \sqsubseteq_{\beta} u_{j}$ because $\left(u_{j}\right)_{b}$ is $\in$-definable from $u_{j}$ and $b \in \mathbb{S}_{\beta}$, and $u_{j} \sqsubseteq_{\beta}\left(u_{j}\right)_{b}$ because $u_{j}=\overline{\pi_{2}}\left(\left(u_{j}\right)_{b}\right)$.

From these observations it follows that $\left\langle\left(u_{i}\right)_{b}, \ldots,\left(u_{\nu}\right)_{b}\right\rangle$ is the $\beta$-pedigree for $\langle b, a\rangle$ over $B \times A$.

Proposition 2.4 (ZFC) Let $V \in \boldsymbol{\beta}_{1} B \backslash \boldsymbol{\beta}_{0} B$; for every $U \in \boldsymbol{\beta}_{\xi} A$ there is a unique $W \in \boldsymbol{\beta}_{\xi+1}(B \times A)$ such that $\left\{U_{y}: y \in B\right\} \in W$ and $\overline{\pi_{1}}(W)=V, \overline{\pi_{2}}(W)=U$. We denote this unique $W$ by $U^{\bowtie} V$.

Proof Assume $U \in \boldsymbol{\beta}_{\xi} A \backslash \boldsymbol{\beta}_{<\xi} A$ and let $\tau_{U}: B \rightarrow \beta_{\xi}(B \times A)$ be defined by $\tau_{U}(y)=U_{y}$.

Existence: We let $W:=\tau_{U}[V]$. As $\tau_{U}$ is one-one and $\operatorname{rank}\left(U_{y}\right)=\operatorname{rank} U$ for all $y \in B$, clearly $W \in \boldsymbol{\beta}_{\xi+1}(B \times A) \backslash \boldsymbol{\beta}_{\xi}(B \times A)$. If $Y \in V$, then $\tau_{U}[Y] \in W$ and $Y=\pi_{1}\left[\tau_{U}[Y]\right] \in \pi_{1}[W]$. Both $V$ and $\pi_{1}[W]$ are ultrafilters over $B$, so $V=\pi_{1}[W]$ and $V=\mathfrak{m}(V)=\mathfrak{m}\left(\overline{\pi_{1}}[W]\right)=\overline{\pi_{1}}(W)$.

$\overline{\pi_{2}}[W]$ is generated by sets of the form $\overline{\pi_{2}}\left[\tau_{U}[Y]\right]$ where $Y \in V$. But $\overline{\pi_{2}}\left[\tau_{U}[Y]\right]=$ $\left\{\overline{\pi_{2}}\left(U_{y}\right): y \in Y\right\}=\{U: y \in Y\}=\{U\}$. So $\overline{\pi_{2}}[W]$ is a principal ultrafilter generated by $U$ and $\overline{\pi_{2}}(W)=\mathfrak{m}\left(\overline{\pi_{2}}[W]\right)=U$.

Uniqueness: Let $W$ have the required properties. The map $\tau_{U}$ is one-one, so $\tau_{U}^{-1}\left(U_{y}\right)=$ $y=\overline{\pi_{1}}\left(U_{y}\right)$; ie, $W$-almost everywhere $\overline{\pi_{1}}=\tau_{U}^{-1}$. If $\overline{\pi_{1}}(W)=V$, then $\overline{\pi_{1}}[W]=V$ as $\left.\left\{U_{y}: y \in B\right\} \in W\right]$, so $\tau_{U}^{-1}[W]=V$, and $W=\tau_{U}[V]$. 
Lemma 2.5 $\left\langle u_{i}^{\bowtie} V,\left(u_{i}\right)_{b},\left(u_{i+1}\right)_{b}, \ldots,\left(u_{\nu}\right)_{b}\right\rangle$ is the $u_{i}$-pedigree for $\langle b, a\rangle$ over $B \times A$.

Proof By Lemma 2.3, $\left\langle\left(u_{i}\right)_{b}, \ldots,\left(u_{\nu}\right)_{b}\right\rangle$ is a $\beta$-pedigree for $\langle b, a\rangle$ over $B \times A$. By the choice of $b, b \mathbb{M}_{\gamma} V$ holds for all $u_{i} \sqsubseteq \gamma \sqsubset \beta$. Hence $\left(u_{i}\right)_{b}=\tau_{u_{i}}(b) \mathbb{M}_{\gamma} \tau_{u_{i}}[V]=u_{i}^{\bowtie} V$ holds for all such $\gamma$ [see the proof of Proposition 2.4]. As $u_{i}^{\bowtie} V \boxminus u_{i}$ and $\left(u_{i}\right)_{b} \boxminus b \boxminus \beta$, $\left\langle u_{i}^{\bowtie} V,\left(u_{i}\right)_{b}, \ldots,\left(u_{\nu}\right)_{b}\right\rangle$ is a $u_{i}$-pedigree.

We can now complete the proof of Proposition 2.2.

Proof of Proposition 2.2 By Lemma 2.5 and RST, Corollary 10.6, the pedigree $\vec{v}$ for $\langle b, a\rangle$ over $B \times A$ has the form $\left\langle v_{0}, \ldots, v_{m},\left(u_{i}\right)_{b}, \ldots,\left(u_{\nu}\right)_{b}\right\rangle$ where $v_{m}=u_{i}^{\bowtie} V$. For $b^{\prime} \boxminus \beta^{\prime}$ such that $u_{i} \sqsubset \beta^{\prime} \sqsubset u_{i+1}\left[u_{\nu} \sqsubset \beta^{\prime}\right.$ if $\left.i=\nu\right]$ and $b^{\prime} \mathbf{M}_{0} V$, the pedigree for $\left\langle b^{\prime}, a\right\rangle$ over $B \times A$ has the form $\vec{v}^{\prime}=\left\langle v_{0}^{\prime}, \ldots, v_{m^{\prime}}^{\prime},\left(u_{i}\right)_{b^{\prime}}, \ldots,\left(u_{\nu}\right)_{b^{\prime}}\right\rangle$ where $v_{m^{\prime}}^{\prime}=u_{i}^{\bowtie} V$. However, it is trivial to verify that $\left\langle v_{0}, \ldots, v_{m},\left(u_{i}\right)_{b^{\prime}}, \ldots,\left(u_{\nu}\right)_{b^{\prime}}\right\rangle$ is also a pedigree for $\left\langle b^{\prime}, a\right\rangle$ over $B \times A$. By the uniqueness of pedigrees [RST, Corollary 10.6], $m=m^{\prime}$ and $v_{j}^{\prime}=v_{j}$ for all $j \leq m$.

Proof of Theorem 2.1 (Strong Stability) It suffices to give the proof for the case $\alpha \boxminus 0$; the general case follows by Transfer.

Let $L:=\operatorname{ran} \vec{u}$ where $\vec{u}=\left\langle u_{0}, \ldots, u_{\nu}\right\rangle$ is the pedigree for $\langle\bar{y}\rangle \in A$ over some $A \in \mathbb{S}_{0}$, and let $\beta \cong_{L} \beta^{\prime}$. If $\beta \boxminus \beta^{\prime} \boxminus u_{i}$ for some $i \leq \nu$, then $\mathcal{P}(\bar{x}, \bar{y} ; \beta) \leftrightarrow \mathcal{P}\left(\bar{x}, \bar{y} ; u_{i}\right) \leftrightarrow$ $\mathcal{P}\left(\bar{x}, \bar{y} ; \beta^{\prime}\right)$, as the variable $z$ appears in $\mathcal{P}(\bar{x}, \bar{y} ; z)$ only in the scope of $\sqsubseteq$ [RST, Definition 12.25].

Assume now that $u_{i} \sqsubset \beta, \beta^{\prime} \sqsubset u_{i+1}$ for some $i \leq \nu\left[u_{i} \sqsubset \beta, \beta^{\prime}\right.$ if $\left.i=\nu\right]$. Fix $V, b \in \mathbb{S}_{0}, V \in \boldsymbol{\beta}_{1} B \backslash \boldsymbol{\beta}_{0} B$, and $b, b^{\prime}$ such that $b \boxminus \beta, b \mathbf{M}_{0} V$ and $b^{\prime} \boxminus \beta^{\prime}, b^{\prime} \mathbf{M}_{0} V$. By Proposition 2.2, the pedigrees for $\langle b,\langle\bar{y}\rangle\rangle$ and $\left\langle b^{\prime},\langle\bar{y}\rangle\right\rangle$ have the form, respectively, $\left\langle v_{0}, \ldots, v_{m},\left(u_{i}\right)_{b}, \ldots,\left(u_{\nu}\right)_{b}\right\rangle$ and $\left\langle v_{0}, \ldots, v_{m},\left(u_{i}\right)_{b^{\prime}}, \ldots,\left(u_{\nu}\right)_{b^{\prime}}\right\rangle$.

To obtain the pedigrees for $\langle\langle\bar{x}\rangle,\langle b,\langle\bar{y}\rangle\rangle\rangle$ and $\left\langle\langle\bar{x}\rangle,\left\langle b^{\prime},\langle\bar{y}\rangle\right\rangle\right\rangle$ where $\langle\bar{x}\rangle \in \mathbb{S}_{0}$, it is only necessary to subscript all terms of the above pedigrees by $\langle\bar{x}\rangle$ [Lemma 2.3]; in particular, both $\langle\langle\bar{x}\rangle,\langle b,\langle\bar{y}\rangle\rangle\rangle$ and $\left\langle\langle\bar{x}\rangle,\left\langle b^{\prime},\langle\bar{y}\rangle\right\rangle\right\rangle$ have the same type $\left(v_{0}\right)_{\langle\bar{x}\rangle}$. It follows that $\langle\bar{x}, \bar{y}, b\rangle$ and $\left\langle\bar{x}, \bar{y}, b^{\prime}\right\rangle$ have the same type. [Apply RST, Theorem 10.10 to the natural mapping $\pi:\langle\langle\bar{x}\rangle,\langle z,\langle\bar{y}\rangle\rangle\rangle \mapsto\langle\bar{x}, \bar{y}, z\rangle$ and its inverse.]

By RST, Theorem 12.11 (Normal Form Theorem), $\mathcal{P}(\bar{x}, \bar{y} ; b) \leftrightarrow \mathcal{P}\left(\bar{x}, \bar{y} ; b^{\prime}\right)$. As the variable $z$ appears in the formula $\mathcal{P}(\bar{x}, \bar{y} ; z)$ only in the scope of $\sqsubseteq$, we have also $\mathcal{P}(\bar{x}, \bar{y} ; \beta) \leftrightarrow \mathcal{P}(\bar{x}, \bar{y} ; b)$ and $\mathcal{P}\left(\bar{x}, \bar{y} ; \beta^{\prime}\right) \leftrightarrow \mathcal{P}\left(\bar{x}, \bar{y} ; b^{\prime}\right)$. This proves the theorem.

Stability for several levels simultaneously is expressed by Polytransfer [RST, Proposition 12.26; RST2, Proposition 1.10 (8)]. The argument in the previous proof can be pushed to establish a version of Strong Stability that generalizes Polytransfer. 
Theorem 2.6 (Strong Stability for several levels) Given $y_{1}, \ldots, y_{\ell}$, there is a level set $L$ such that if $\alpha \sqsubset \beta_{1} \sqsubset \ldots \sqsubset \beta_{n}, \alpha \sqsubset \beta_{1}^{\prime} \sqsubset \ldots \sqsubset \beta_{n}^{\prime}$, and $\beta_{i} \cong_{L} \beta_{j}^{\prime}$ for all $i, j \leq n$, then

$$
\left(\forall \bar{x} \in \mathbb{S}_{\alpha}\right)\left(\mathcal{P}^{\alpha}\left(\bar{x}, \bar{y} ; \beta_{1}, \ldots, \beta_{n}\right) \leftrightarrow \mathcal{P}^{\alpha}\left(\bar{x}, \bar{y} ; \beta_{1}^{\prime}, \ldots, \beta_{n}^{\prime}\right)\right) .
$$

Proof We give the proof for $\alpha \boxminus 0$ and $n=2$. Using the notation from the proof of Theorem 2.1, let $u_{i} \sqsubset \beta_{1}, \beta_{2}, \beta_{1}^{\prime}, \beta_{2}^{\prime} \sqsubset u_{i+1}$. Let $b_{i} \boxminus \beta_{i}, b_{i} \mathbf{M}_{0} V, b_{i}^{\prime} \boxminus \beta_{i}^{\prime}, b_{i}^{\prime} \mathbf{M}_{0} V$, for $i=1,2$. By Proposition 2.2 applied twice, the pedigree for $\left\langle b_{1},\left\langle b_{2},\langle\bar{y}\rangle\right\rangle\right\rangle$ then has the form

$$
\left\langle v_{0}, \ldots, v_{m},\left(u_{i}^{\bowtie} V\right)_{b_{1}},\left(\left(u_{i}\right)_{b_{2}}\right)_{b_{1}}, \ldots,\left(\left(u_{\nu}\right)_{b_{2}}\right)_{b_{1}}\right\rangle
$$

and $v_{0}, \ldots, v_{m}=\left(u_{i}^{\bowtie} V\right)^{\bowtie} V$ are independent of the choice of $\beta_{1}, \beta_{2}, b_{1}, b_{2}$. It follows that $\left\langle\langle\bar{x}\rangle,\left\langle b_{1},\left\langle b_{2},\langle\bar{y}\rangle\right\rangle\right\rangle\right\rangle$ and $\left\langle\langle\bar{x}\rangle,\left\langle b_{1}^{\prime},\left\langle b_{2}^{\prime},\langle\bar{y}\rangle\right\rangle\right\rangle\right\rangle$ have the same type, namely $\left(v_{0}\right)_{\langle\bar{x}\rangle}$. The theorem follows from this observation.

Theorem 2.6 suggests a further generalization, in which the condition " $\beta_{i} \cong_{L} \beta_{j}^{\prime}$ for all $i, j \leq n$ " would be weakened to " $\beta_{i} \cong_{L} \beta_{i}^{\prime}$ for all $i \leq n$." However, such generalization is false. Below, we give a counterexample.

First we need some observations. The technical notion of $\operatorname{good} \alpha$-pedigree is defined in RST, Definition 10.8. The following fact is used implicitly in RST.

Proposition 2.7 (SST ${ }^{b}$; hence GRIST) Every $\alpha$-pedigree is good.

Proof The axiom $\left(\mathbf{B}_{\alpha}\right)$ [RST, page 65] implies that for every $y \in B \in \mathbb{S}_{\alpha}$ there is a good $\alpha$-pedigree $\vec{v}$ for $y$ over $B$. Indeed, fix $x \in A \in \mathbb{S}_{\alpha}$ where $x \in \mathbb{S}_{\alpha}$; then $\langle x\rangle$ is a good $\alpha$-pedigree for $U=x$ over $A$, Dom $U=A$, and $x \mathbf{M}_{\alpha} U$ holds. Let $F: B \rightarrow A$ be the constant function with value $x$; so $F \in \mathbb{S}_{\alpha}$ and $\operatorname{ran} F \subseteq \operatorname{Dom} U$. By $\left(\mathbf{B}_{\alpha}\right)$ there is $V \in \mathbb{S}_{\alpha}$ with $U=\bar{F}(V)$ and $y \mathbf{M}_{\alpha} V$. The last statement asserts that there is a good $\alpha$-pedigree $\vec{v}=\left\langle v_{0}, \ldots, v_{\mu}\right\rangle$ for $y$ over some $B^{\prime} \in \mathbb{S}_{\alpha}$ with $v_{0}=V$. But, $\bar{F}(V)$ being defined implies Dom $V=B$; as Dom $v_{0}=B^{\prime}$, we have $B=B^{\prime}$. If now $\vec{u}$ is any $\alpha$-pedigree for $y$ over $B$, then $\vec{u}=\vec{v}$ by RST, Proposition 10.3 , so $\vec{u}$ is good.

Proposition 2.8 If $\vec{u}$ is an $\alpha$-pedigree (for $x$ over $A \in \mathbb{S}_{\alpha}$ ), then

$$
\left(\mathbb{S}_{\alpha}[\vec{u}],=, \in, \sqsubseteq_{\alpha}\right) \vDash(\forall w)(\exists k \in \omega)\left(w \boxminus u_{k}\right) .
$$

Proof The claim follows from RST, Proposition 10.20. By RST, Definition 10.8 (and the fact that, in GRIST, all pedigrees are good), the interpretation $[\mathcal{U} \ell t(\mathbb{V} ; \Sigma \mathbf{u})]^{\mathbb{S}_{\alpha}}$ is 
isomorphic to $\mathbb{S}_{\alpha}[\vec{u}]$ via $\mathfrak{j}=\mathfrak{j}_{\alpha ; x, A}$, and $\mathfrak{j}(D)=D\left(\vec{u}^{+}\right)=\vec{u}^{+}, \mathfrak{j}(E)=E\left(\vec{u}^{+}\right)=x$, in the notation of RST, Proposition 10.20. By the same proposition (valid in ZFC),

$$
\mathcal{U} \ell t(\mathbb{V} ; \Sigma \mathbf{u}) \vDash(\forall f \sqsupset 0)(\exists k \in \omega)\left(f \boxminus D_{k}\right) .
$$

As $\mathbb{S}_{\alpha} \preccurlyeq \mathbb{I}$, the same is true in $[\mathcal{U} \ell t(\mathbb{V} ; \Sigma \mathbf{u})]^{\mathbb{S}_{\alpha}}$, and via the isomorphism $\mathfrak{j}$, in $\mathbb{S}_{\alpha}[\vec{u}]$. Hence

$$
\mathbb{S}_{\alpha}[\vec{u}] \vDash(\forall w \sqsupset 0)(\exists k \in \omega)\left(w \boxminus\left(\vec{u}^{+}\right)_{k}=u_{k+1}\right) .
$$

Of course, $\mathbb{S}_{\alpha}[\vec{u}] \vDash w \boxminus u_{0}$, if $w \boxminus 0$.

Note: In RST, the notations $\mathcal{U} \ell t(\mathbb{V} ; \mathcal{U})$ and $\mathcal{U} \ell t(\mathbb{V} ; \Sigma \mathcal{U})$ are used interchangeably.

Proposition 2.9 If $\vec{u}=\left\langle u_{0}, \ldots, u_{\nu}\right\rangle$ is an $\alpha$-pedigree and $\nu \in \mathbb{S}_{\alpha}$, then rank $u_{0}=\nu$.

Proof It suffices to give a proof for $\alpha \boxminus 0$ [then use Transfer]. We prove that rank $u_{\nu-i}=i$ by External Induction [RST, Proposition 12.19]. If $i=0$, then $u_{\nu-i}=$ $u_{\nu}=a \in \boldsymbol{\beta}_{0} A$. If $u_{\nu-i} \in \boldsymbol{\beta}_{i} A \backslash \boldsymbol{\beta}_{i-1} A$, then $u_{\nu-(i+1)}$ is nonprincipal and generated by $u_{\nu-i}$ over $\boldsymbol{\beta}_{i} A$, so it belongs to $\boldsymbol{\beta}_{i+1} A \backslash \boldsymbol{\beta}_{i} A$.

\section{Construction of the example.}

Let $A \in \mathbb{S}_{0}$ be an infinite set and $U \in \boldsymbol{\beta}_{\omega} A \backslash \boldsymbol{\beta}_{<\omega} A$ be a stratified ultrafilter over $A$ of rank $\omega$. The axiom (F) [RST, page 66] guarantees that there is an $a \in A$ such that $a \mathbf{M}_{0} U$ holds. We fix such an $a$ and $V, B \in \mathbb{S}_{0}, V \in \boldsymbol{\beta}_{1} B \backslash \boldsymbol{\beta}_{0} B$.

Consider the statement $\mathcal{P}\left(A, V, B, a ; \beta_{1}, \beta_{2}\right)$ :

"For every $b \in B$ such that $b \boxminus \beta_{2}$ and $b \mathbf{M}_{0} V$, and for every pedigree $\vec{v}=\left\langle v_{j}: j \leq \mu\right\rangle$ for $\langle b, a\rangle$ over $B \times A$, there exists some $j \leq \mu$ such that $v_{j} \boxminus \beta_{1}$."

Let $L=\left\{w_{0}, w_{1}, \ldots, w_{\rho}\right\}$ be any level set; wlog $w_{0} \boxminus 0$. We find $\beta_{1} \cong_{L} \beta_{1}^{\prime}$ and $\beta_{2}$ such that $\mathcal{P}\left(A, V, B, a ; \beta_{1}, \beta_{2}\right)$ is true and $\mathcal{P}\left(A, V, B, a ; \beta_{1}^{\prime}, \beta_{2}\right)$ is false.

Let $\vec{u}=\left\langle u_{0}, u_{1}, \ldots, u_{\nu}\right\rangle$ be the pedigree for $a$ over $A$; we have $u_{0}=U$. We note that $\nu \notin \mathbb{S}_{0} ;$ otherwise $U$ would have rank $\nu \in \omega$, by Proposition 2.9.

Fix a level $\gamma$ such that $0 \sqsubset \gamma \sqsubset u_{1}, w_{1}$. Fix $W \in \boldsymbol{\beta}_{1} \omega \backslash \boldsymbol{\beta}_{0} \omega$ and $c \boxminus \gamma, c \mathbf{M}_{0} W$. Then $c \in \omega$ and $c \notin \mathbb{S}_{0}$ because $W$ is nonprincipal. We claim that $c<\nu$. Assume to the contrary that $\nu \leq c$, so $\nu \in \mathbb{S}_{\gamma}$. As $\left\langle u_{0}, \ldots, u_{\nu}\right\rangle$ is also a $\gamma$-pedigree for $a$ over $A$, by Proposition $2.9 \operatorname{rank} U=\operatorname{rank} u_{0}=\nu$ and $U \in \boldsymbol{\beta}_{<\omega} A$, a contradiction.

Fix $\beta_{2}$ such that $u_{c} \sqsubset \beta_{2} \sqsubset u_{c+1}$. We observe that $b, a, \vec{u} \in \mathbb{S}_{0}[\vec{v}]$ [the last by RST, Corollary 10.18] and $c$ is $\in$-definable from $\vec{u}, \vec{v}$ and $b: c$ is the least $j$ such that 
$\left(u_{j}\right)_{b}=v_{k}$ for some $k \leq \mu$. Hence $c \in \mathbb{S}_{0}[\vec{v}]$ and, by Proposition 2.8, $c \boxminus v_{\ell}$ for some $\ell>0$.

The statement $\mathcal{P}\left(A, V, B, a ; \beta_{1}, \beta_{2}\right)$ holds for $\beta_{1}:=\gamma$ and $\beta_{2}$, because then $\beta_{1} \boxminus c \boxminus v_{\ell}$.

On the other hand, by density of levels, there exist $\beta_{1}^{\prime}$ such that $0 \sqsubset \beta_{1}^{\prime} \sqsubset v_{1} \sqsubseteq$ $v_{\ell} \boxminus \gamma \sqsubset w_{1}$. Then $\beta_{1}^{\prime} \cong_{L} \beta_{1}$ and $\mathcal{P}\left(A, V, B, a ; \beta_{1}^{\prime}, \beta_{2}\right)$ fails.

This example can also be used to show that Polytransfer [RST, Proposition 12.26] does not hold for arbitrary finite (in the sense of GRIST) sequences of levels.

We say that sets $S_{1}$ and $S_{2}$ are level-equivalent if $\left(\forall x \in S_{1}\right)\left(\exists y \in S_{2}\right)(x \boxminus y)$ and $\left(\forall y \in S_{2}\right)\left(\exists x \in S_{1}\right)(x \boxminus y)$.

Claim For A, U, a as above, the length $\nu$ of the pedigree $\vec{u}$ for a over $A$ has itself a pedigree of the form $\left\langle v_{0}, v_{1}\right\rangle$, where $v_{1} \boxminus u_{1}$.

Proof Refer to RST, Proposition 10.20. The interpretation $\left(\mathbb{S}_{0}[\vec{u}],=, \in, \sqsubseteq\right)$ is isomorphic to $[\mathcal{U} \ell t(\mathbb{V} ; \Sigma \mathbf{U})]^{\mathbb{S}_{0}}$, and $\vec{v}^{+}$corresponds to $D$ in this isomorphism. This means that $\nu$ corresponds to the function $F: t \mapsto|t|$ defined for $t \in \Sigma \mathbf{U}$. Each such $t$ has the form $\langle u\rangle \frown s$, for some $u \in U$. As the rank of $U$ is $\omega$, $\operatorname{rank} u \in \omega$, and hence $|s|=\operatorname{rank} u$ is independent of $s$. Define $f:\left[T_{\mathbf{U}}\right]_{0}=U \rightarrow \omega$ by $f(u)=\operatorname{rank} u$; the above remarks show that $F(t)=f(u)$ holds for all $t$, ie, $\mathcal{U} \ell t(\mathbb{V} ; \Sigma \mathbf{U}) \vDash F \boxminus D^{1}$. Hence $\mathcal{U} \ell t(\mathbb{V} ; \Sigma \mathbf{U})$ satisfies the statement "The range of the pedigree for $F$ is level-equivalent to $\left\{D^{0}, D^{1}\right\} . "$ As $\mathbb{S}_{\alpha} \preccurlyeq \mathbb{I}$, the same is true in $[\mathcal{U} \ell t(\mathbb{V} ; \Sigma \mathbf{U})]^{\mathbb{S}_{0}}$. The isomorphism then establishes that $\mathbb{S}_{0}[\vec{u}]$ satisfies the statement "The range of the pedigree for $\nu$ is level-equivalent to $\left\{u_{0}, u_{1}\right\}$," and by RST, Proposition 10.17, the range of the pedigree for $\nu$ is levelequivalent to $\left\{u_{0}, u_{1}\right\}$.

Let now $\Lambda_{1}=\left\{u_{0} \sqsubset u_{1} \sqsubset \ldots \sqsubset u_{\nu-1}\right\}$ and $\Lambda_{2}=\left\{u_{0} \sqsubset u_{2} \sqsubset \ldots \sqsubset u_{\nu}\right\}$. The statement: "There is a pedigree $\vec{v}$ such that ran $\vec{v}$ is level-equivalent to $\Lambda$ " is true about $\Lambda_{1}\left[\left\langle u_{0}, u_{1}, \ldots, u_{\nu-1}\right\rangle\right.$ is a pedigree for $\left.u_{\nu-1} \in \boldsymbol{\beta}_{1} A \in \mathbb{S}_{0}\right]$, but false about $\Lambda_{2}$ [there can be no such pedigree, because $\nu$ would be $\in$-definable from it, and the range of the pedigree for $\nu$ is level-equivalent to $\left\{u_{0}, u_{1}\right\}$; but $u_{1} \boxminus u_{j}$ does not hold for any $j \neq 1$, contradicting Proposition 2.8 and RST, Proposition 10.17].

A weaker generalization of Theorem 2.6 does hold. We state it only for $n=2$; the higher values of $n$ can be treated similarly.

Theorem 2.10 Given $y_{1}, \ldots, y_{\ell}$, there is a level set $L$ such that for every $\beta_{2}$ there is a level set $M$, dependent only on the $\cong_{L}$-equivalence class of $\beta_{2}$, such that if $\alpha \sqsubset \beta_{1} \sqsubset \beta_{2}, \alpha \sqsubset \beta_{1}^{\prime} \sqsubset \beta_{2}^{\prime}, \beta_{2} \cong_{L} \beta_{2}^{\prime}$ and $\beta_{1} \cong_{M} \beta_{1}^{\prime}$, then

$$
\left(\forall \bar{x} \in \mathbb{S}_{\alpha}\right)\left(\mathcal{P}^{\alpha}\left(\bar{x}, \bar{y} ; \beta_{1}, \beta_{2}\right) \leftrightarrow \mathcal{P}^{\alpha}\left(\bar{x}, \bar{y} ; \beta_{1}^{\prime}, \beta_{2}^{\prime}\right)\right)
$$


Proof Choose $V, b_{1}, b_{2}, b_{1}^{\prime}, b_{2}^{\prime}$ as in the proof of Theorem 2.6 (let $n=2$ ). The pedigree for $\left\langle b_{2},\langle\bar{y}\rangle\right\rangle$ is $\left\langle v_{0}, \ldots, v_{m},\left(u_{i}\right)_{b_{2}}, \ldots,\left(u_{\nu}\right)_{b_{2}}\right\rangle$, where $v_{m}=u_{i}^{\bowtie} V$, so $v_{m} \boxminus u_{i}$, and $v_{0}, \ldots, v_{m}$ depend only on the $\cong_{L}$-equivalence class of $\beta_{2}$. Let $M:=\left\{v_{0}, \ldots, v_{m}\right\}$.

If $v_{j} \sqsubset \beta_{1} \sqsubset v_{j+1}\left[v_{j} \sqsubset \beta_{1}\right.$ if $\left.j=m\right]$, the pedigree for $\left\langle b_{1},\left\langle b_{2},\langle\bar{y}\rangle\right\rangle\right\rangle$ is

$$
\left\langle w_{0}, \ldots, w_{p},\left(v_{j}\right)_{b_{1}},\left(v_{j+1}\right)_{b_{1}}, \ldots,\left(v_{m}\right)_{b_{1}},\left(\left(u_{i}\right)_{b_{2}}\right)_{b_{1}}, \ldots,\left(\left(u_{\nu}\right)_{b_{2}}\right)_{b_{1}}\right\rangle,
$$

where $w_{p}=v_{j}^{\bowtie} V$, so $w_{p} \boxminus v_{j}$, and $w_{0}, \ldots, w_{p}$ are independent of the choice of $b_{1}, b_{2}$. Hence $\left\langle\langle\bar{x}\rangle,\left\langle b_{1},\left\langle b_{2},\langle\bar{y}\rangle\right\rangle\right\rangle\right\rangle$ and $\left\langle\langle\bar{x}\rangle,\left\langle b_{1}^{\prime},\left\langle b_{2}^{\prime},\langle\bar{y}\rangle\right\rangle\right\rangle\right\rangle$ have the same type $\left(w_{0}\right)_{\langle\bar{x}\rangle}$, and the theorem follows.

\section{Miscellaneous other principles.}

In this section we derive, in GRIST, several versions of Idealization and Choice that have been found useful in applications. In this section, $\mathcal{P}$ is always a $\mathbf{V}$-formula, unless stated otherwise.

Local Idealization combines Idealization and Local Transfer.

\section{Local FRIST Idealization:}

Given $\mathbf{U}$ and $x_{1}, \ldots, x_{n}$, there is $\mathbf{V} \supset \mathbf{U}$ such that for all $A, B \in \mathbf{U}$,

$\left(\forall a \in \mathcal{P}^{\text {fin }} A \cap \mathbf{U}\right)(\exists y \in B)(\forall x \in a) \mathcal{P}(x, y, \bar{x} ; \mathbf{U}) \leftrightarrow(\exists y \in B)(\forall x \in A \cap \mathbf{U}) \mathcal{P}(x, y, \bar{x} ; \mathbf{V})$

Proof By Local Transfer there is $\mathbf{V} \supset \mathbf{U}$ such that for all $A, B \in \mathbf{U}$ and all $a \in \mathbf{U}$,

$$
(\exists y \in B)(\forall x \in a) \mathcal{P}(x, y, \bar{x} ; \mathbf{U}) \leftrightarrow(\exists y \in B)(\forall x \in a) \mathcal{P}(x, y, \bar{x} ; \mathbf{V}) .
$$

Hence the left side of the claim is equivalent to

$$
\left(\forall a \in \mathcal{P}^{\text {fin }} A \cap \mathbf{U}\right)(\exists y \in B)(\forall x \in a) \mathcal{P}(x, y, \bar{x} ; \mathbf{V}),
$$

which is equivalent to the right side by FRIST Idealization.

\section{Local GRIST Idealization:}

Given $\mathbf{U}$ and $x_{1}, \ldots, x_{n}$, there is $\mathbf{V} \supset \mathbf{U}$ such that, for all $A \in \mathbf{U}$,

$\left(\forall a \in \mathcal{P}^{\text {fin }} A \cap \mathbf{U}\right)(\exists y)(\forall x \in a) \mathcal{P}(x, y, \bar{x} ; \mathbf{U}) \leftrightarrow(\exists y)(\forall \mathbf{W} \subset \mathbf{V})(\forall x \in A \cap \mathbf{W}) \mathcal{P}(x, y, \bar{x} ; \mathbf{V})$ 
Proof By Local Transfer there is $\mathbf{V} \supset \mathbf{U}$ such that, for all $A \in \mathbf{U}$,

$\left(\forall a \in \mathcal{P}^{\mathbf{f i n}} A \cap \mathbf{U}\right)(\exists y)(\forall x \in a) \mathcal{P}(x, y, \bar{x} ; \mathbf{U}) \leftrightarrow\left(\forall a \in \mathcal{P}^{\mathrm{fin}} A \cap \mathbf{V}\right)(\exists y)(\forall x \in a) \mathcal{P}(x, y, \bar{x} ; \mathbf{V})$ and, for all finite $a \in \mathbf{U}$,

$$
(\exists y)(\forall x \in a) \mathcal{P}(x, y, \bar{x} ; \mathbf{U}) \leftrightarrow(\exists y)(\forall x \in a) \mathcal{P}(x, y, \bar{x} ; \mathbf{V}) .
$$

Assume that $\left(\forall a \in \mathcal{P}^{\text {fin }} A \cap \mathbf{U}\right)(\exists y)(\forall x \in a) \mathcal{P}(x, y, \bar{x} ; \mathbf{U})$. Then in particular

$$
(\forall \mathbf{W} \subset \mathbf{V})\left(\forall a \in \mathcal{P}^{\mathrm{fin}} A \cap \mathbf{W}\right)(\exists y)(\forall x \in a) \mathcal{P}(x, y, \bar{x} ; \mathbf{V}),
$$

which is the left side of GRIST Idealization. The right side follows.

Assume now that $(\exists y)(\forall \mathbf{W} \subset \mathbf{V})(\forall x \in A \cap \mathbf{W}) \mathcal{P}(x, y, \bar{x} ; \mathbf{V})$, and let $\mathbf{W}=\mathbf{U}$ to obtain $(\exists y)(\forall x \in A \cap \mathbf{U}) \mathcal{P}(x, y, \bar{x} ; \mathbf{V})$. It follows that $(\exists y)(\forall x \in a) \mathcal{P}(x, y, \bar{x} ; \mathbf{V})$ holds for all $a \in \mathcal{P}^{\text {fin }} A \cap \mathbf{U}$ [because $a \subseteq A$, by RST2, Proposition 1.10 (13)], and hence $(\exists y)(\forall x \in a) \mathcal{P}(x, y, \bar{x} ; \mathbf{U})$ holds.

We recall the principle of Standard Size Choice [RST2, Proposition 1.10 (10)]:

For every $A \in \mathbf{V}$ such that $(\forall x \in A \cap \mathbf{V})(\exists y) \mathcal{P}(x, y, \bar{x} ; \mathbf{V})$, there exists a function $f$ with $\operatorname{dom} f=A$ such that $(\forall x \in A \cap \mathbf{V}) \mathcal{P}(x, f(x), \bar{x} ; \mathbf{V})$.

We call $f$ a choice function for $\mathcal{P}$ on $A$.

Strong Standard Size Choice: For every $\mathbf{V}=\mathbf{V}(\alpha)$ there is $\mathbf{V}^{\prime} \supset \mathbf{V}$ such that for all $A \in \mathbf{V}$ there exists a function $f$ with $\operatorname{dom} f=A$ such that, for all $x \in A$ with $\mathbf{V}(x) \subset \mathbf{V}^{\prime}, \quad(\exists y) \mathcal{P}(x, y, \bar{x} ; \mathbf{V}(\alpha, x)) \rightarrow \mathcal{P}(x, f(x), \bar{x} ; \mathbf{V}(\alpha, x))$.

The level $\mathbf{V}^{\prime}$ depends on the parameters $\bar{x}$. If $\mathbf{V}^{\prime}$ were allowed to depend also on $f$, the principle would follow easily from Standard Size Choice and Local Transfer. To prove it as is, we follow the argument of RST, Proposition 12.28 ( $\alpha$-Standard Size Choice) with minor changes, except for the proof of Claim, where the use of Local Transfer has to be replaced by an appeal to Strong Stability.

Proof We first reformulate Strong Standard Size Choice in the $\epsilon-\sqsubseteq-$ language.

Let $\mathcal{P}(x, y, \bar{x})$ be an $\in$ - $\sqsubseteq$-formula. For every $\alpha$ there is $\beta \sqsupset \alpha$ such that for all $A \sqsubseteq \alpha$ there is a function $f$ with $\operatorname{dom} f \subseteq A$ such that, for all $x \in A, x \sqsubset \beta$, $(\exists y) \mathcal{P}^{\langle\alpha, x\rangle}(x, y, \bar{x}) \rightarrow \mathcal{P}^{\langle\alpha, x\rangle}(x, f(x), \bar{x})$.

We fix $\bar{x}$ and $\alpha$. Let $\mathcal{P}^{\prime}(z, y)$ be the formula $(\exists x, \bar{x})[z=\langle x,\langle\bar{x}\rangle\rangle \wedge \mathcal{P}(x, y, \bar{x})]$, and let $\mathcal{Q}(V)$ be the $\in$-formula corresponding to $\mathcal{P}^{\prime}$ by the Normal Form Theorem. If $\alpha \sqsubseteq \gamma,\langle\bar{x}\rangle \mathbf{M}_{\gamma} U$ and $x \in \mathbb{S}_{\gamma}$, we then have

$$
(\exists y) \mathcal{P}^{\gamma}(x, y, \bar{x}) \leftrightarrow(\exists V)\left[\overline{\pi_{1}}(V)=U_{x} \wedge \mathcal{Q}(V)\right] .
$$


The ZFC principle of Selection: Let $\mathcal{R}(x, y, \bar{p})$ be an $\in$-formula;

$(\forall \bar{p})(\forall A)[(\forall x \in A)(\exists y) \mathcal{R}(x, y, \bar{p}) \rightarrow(\exists f)(f$ is a function $\wedge(\forall x \in A) \mathcal{R}(x, f(x), \bar{p}))]$, which holds in $\left(\mathbb{S}_{\alpha}, \in\right)$, implies that for every $A \sqsubseteq \alpha$ there are functions $V, B \in \mathbb{S}_{\alpha}$ such that $\operatorname{dom} V=\operatorname{dom} B=\left\{x \in A:(\exists V)\left[\pi_{1}(V)=U_{x} \wedge \mathcal{Q}(V)\right]\right\}$, and for all $x \in \operatorname{dom} V, V(x) \in \boldsymbol{\beta}_{\infty} B(x) \wedge \overline{\pi_{1}}(V(x))=U_{x} \wedge \mathcal{Q}(V(x))$. It remains to prove the following.

Claim. There is a level $\beta \sqsupset \alpha$, and for every $A \sqsubseteq \alpha$ there is a function $\vec{v}$ with $\operatorname{dom} \vec{v}=\operatorname{dom} V$ such that for all $x \in \operatorname{dom} \vec{v}, x \sqsubset \beta, \vec{v}(x)=\left\langle v(x)_{0}, \ldots, v(x)_{\nu(x)}\right\rangle$ is an $\langle\alpha, x\rangle$-pedigree over $B(x)$ with $v(x)_{0}=V(x)$ and $v(x)_{\nu(x)}=\langle\langle x,\langle\bar{x}\rangle\rangle, y(x)\rangle$ for some (uniquely determined) $y(x)$.

The function $f$ on $A$ defined on $\operatorname{dom} V$ by $x \mapsto y(x)$ then has the property that, for all $x \sqsubset \beta$ such that $(\exists y) \mathcal{P}^{\langle\alpha, x\rangle}(x, y, \bar{x}),\langle\langle x,\langle\bar{x}\rangle\rangle, f(x)\rangle \mathbf{M}_{\langle\alpha, x\rangle} V(x) \wedge \mathcal{Q}(V(x))$; so $\left(\mathcal{P}^{\prime}\right)^{\langle\alpha, x\rangle}(\langle x,\langle\bar{x}\rangle\rangle, f(x))$ holds, ie, $\mathcal{P}^{\langle\alpha, x\rangle}(x, f(x), \bar{x})$ holds.

\section{Proof of Claim.}

Let $\vec{u}=\left\langle u_{0}, u_{1}, \ldots, u_{\mu}\right\rangle$ be an $\alpha$-pedigree for $\langle\bar{x}\rangle$. We fix a level $\beta$ such that $\alpha \sqsubset \beta \sqsubset u_{1}[\alpha \sqsubset \beta$ if $\mu=0]$. Let $x \in \operatorname{dom} V, x \sqsubset \beta$.

By Lemma 2.3, $\vec{u}_{x}:=\left\langle\left(u_{0}\right)_{x},\left(u_{1}\right)_{x}, \ldots,\left(u_{\mu}\right)_{x}\right\rangle$ is an $\langle\alpha, x\rangle$-pedigree for $\langle x,\langle\bar{x}\rangle\rangle$, and $\left(u_{j}\right)_{x} \boxminus_{\langle\alpha, x\rangle} u_{j}$ for all $j \leq \mu$. Let $\vec{v}=\left\langle v_{0}, v_{1}, \ldots, v_{\nu(x)}\right\rangle$ be some $\langle\alpha, x\rangle$-pedigree over $B(x)$ with $v_{0}=V(x)$ and $v_{\nu(x)}=\langle\langle x,\langle\bar{x}\rangle\rangle, y\rangle$, for some $y$. If $\beta \sqsubset v_{1}$ [or if $\mu=0$ ], then $\vec{v}$ is also a $\beta$-pedigree. If $v_{1} \boxminus \gamma \sqsubseteq \beta$, fix $\delta$ so that $\beta \sqsubset \delta \sqsubset u_{1}[\beta \sqsubset \delta$ if $\mu=0$ ] (density). Then $\gamma \cong_{L} \delta$ for $L:=\operatorname{ran} \vec{u}_{x}$, so by Strong Stability, there is an $\langle\alpha, x\rangle$-pedigree $\vec{v}$ as above with $v_{1} \boxminus \delta \sqsupset \beta$. We again conclude that $\vec{v}$ is a $\beta$-pedigree.

The above argument shows that for every $x \in \operatorname{dom} V$ there is a $\beta$-pedigree $\vec{v}$ over $B(x)$ with $v_{0}=V(x)$ and $v_{\nu(x)}=\langle\langle x,\langle\bar{x}\rangle\rangle, y\rangle$ for some $y$.

Similar to the proof of RST, Proposition 12.28, using $\alpha$-Finite Choice and GRIST Idealization we obtain a function $\vec{v}$ with $\operatorname{dom} \vec{v}=\operatorname{dom} V$ such that for every $x \in \operatorname{dom} V$, $x \sqsubset \beta, \vec{v}(x)$ is a $\beta$-pedigree with the required properties. As $v(x)_{0}=V(x) \sqsubseteq\langle\alpha, x\rangle$ and $v(x)_{1} \sqsupset \beta \sqsupset\langle\alpha, x\rangle$ [if $\nu(x)>0$ ], $\vec{v}(x)$ is an $\langle\alpha, x\rangle$-pedigree.

The last principle we consider was proposed by Andreev [1] in the context of IST. It is a strengthening of the well-known Robinson's Lemma. The most important case is $A=\mathbb{N}$. If $\mathcal{Q}(n, y, \bar{z})$ is an internal statement [RST2, page 12; [6]], $F$ is a function on $\mathbb{N}$, and $\mathcal{Q}(n, F(n), \bar{z})$ is valid for all $n \in \mathbf{V}$, then by overflow it remains valid for some, but not necessarily all, $n \notin \mathbf{V}$ as well. Andreev Principle says that we can do better, at least for internal statements with parameters from $\mathbf{V}$. 
Definition 3.1 A function $F$ with $\operatorname{dom} F=A \in \mathbf{V}$ is called adequate relative to $\mathbf{V}$ if $(\forall X \in \mathbf{V})(F\lceil\mathbf{V} \subseteq X \rightarrow F \subseteq X)$.

The usefulness of adequate functions comes from the following observation.

Let $\mathcal{Q}(x, y, \bar{z})$ be any internal formula. If $\bar{z} \in \mathbf{V}$ and $\mathcal{Q}(x, F(x), \bar{z})$ holds for all $x \in A \cap \mathbf{V}$, then it holds for all $x \in A$.

Proof Fix $B \in \mathbf{V}$ such that $\operatorname{ran} F \subseteq B$ and let $X=\{\langle x, y\rangle \in A \times B: \mathcal{Q}(x, y, \bar{z})\}$. Then $X \in \mathbf{V}$ and the definition of adequacy for this $X$ translates into the preceding observation.

Theorem 3.2 For every function $G$ defined on $A \in \mathbf{V}$ there exists an adequate function $F$ defined on $A$ such that $F \uparrow \mathbf{V}=G \uparrow \mathbf{V}$.

Proof Let $G: A \rightarrow B$ be given, with $A, B \in \mathbf{V}$. By FRIST Standardization, there is a set $K \in \mathbf{V}, K \subseteq \mathcal{P}(A \times B)$, such that for all $X \subseteq A \times B, X \in \mathbf{V}$,

$$
X \in K \leftrightarrow(\forall x \in A \cap \mathbf{V})(\langle x, G(x)\rangle \in X) .
$$

We note that $X_{1}, X_{2} \in K \cap \mathbf{V}$ implies $X_{1} \cap X_{2} \in K$, hence, by Transfer, $K$ is closed under finite intersections. Also, $(\forall x \in A)(\exists y)(\langle x, y\rangle \in X)$ holds for all $X \in K \cap \mathbf{V}$, hence, by Transfer, for all $X \in K$.

For every $a \in A$ let $L_{a}=\{\langle x, y\rangle \in A \times B: x=a \rightarrow y=G(a)\}$.

It is now clear that for every finite $\left\{X_{1}, \ldots, X_{n}\right\} \subseteq K \cap \mathbf{V}$ and $\left\{a_{1}, \ldots, a_{m}\right\} \subseteq A \cap \mathbf{V}$ there is a function $F: A \rightarrow B$ such that

$$
F \subseteq X_{1} \cap \ldots \cap X_{n} \cap L_{a_{1}} \cap \ldots \cap L_{a_{m}} .
$$

By Saturation (Idealization) there is $F$ such that $F \subseteq X \cap L_{a}$ for all $X \in K \cap \mathbf{V}$ and $a \in A \cap \mathbf{V}$. Clearly $F$ is adequate and $F \uparrow A=G \uparrow A$.

Corollary 3.3 (Andreev Principle) Every external function defined on $A \cap \mathbf{V}$ has an extension to a function $F$ defined on $A$ such that if $\mathcal{Q}(x, y, \bar{z})$ is any internal formula, $\bar{z} \in \mathbf{V}$ and $\mathcal{Q}(x, F(x), \bar{z})$ holds for all $x \in A \cap \mathbf{V}$, then it holds for all $x \in A$.

\section{A typical application of Andreev Principle.}

Let $\left\langle a_{n}: n \in \mathbb{N}\right\rangle$ be an adequate sequence where each $a_{n} \simeq 0$ relative to $\mathbf{V}$, for $n \in \mathbf{V}$. Then $\Sigma_{n=0}^{\infty}\left|a_{n}\right| \simeq 0$ relative to $\mathbf{V}$.

Proof For any fixed $\varepsilon>0, \varepsilon \in \mathbf{V}$, the statement $\mathcal{Q}(n, a, \varepsilon):|a|<\varepsilon / 2^{n}$ is internal and $\left|a_{n}\right|<\varepsilon / 2^{n}$ holds for all $n \in \mathbb{N} \cap \mathbf{V}$. Hence, $\left|a_{n}\right|<\varepsilon / 2^{n}$ holds for all $n \in \mathbb{N}$. It follows that $\sum_{n=0}^{\infty}\left|a_{n}\right|<2 \varepsilon$. As $\varepsilon \in \mathbf{V}$ is arbitrary, $\sum_{n=0}^{\infty}\left|a_{n}\right| \simeq 0$. 


\section{Relative continuity}

Definition 4.1 (RST2, Definition 2.17) The function $f$ is (uniformly) $\left(\mathbf{V}_{1}, \mathbf{V}_{2}\right.$ )continuous if $x \simeq \mathbf{v}_{1} x^{\prime}$ implies $f(x) \simeq \mathbf{v}_{2} f\left(x^{\prime}\right)$, for all $x, x^{\prime} \in \operatorname{dom} f$. The function $f$ is $\mathbf{V}$-continuous if it is $(\mathbf{V}, \mathbf{V})$-continuous.

RST2, Theorem 2.10 shows that for every function $f$ there is a finite set $\left\{v_{0}, \ldots, v_{n}\right\}$ such that $\mathbf{V}(\cdot)=\mathbf{V}\left(v_{0}\right) \subset \mathbf{V}\left(v_{1}\right) \subset \ldots \subset \mathbf{V}\left(v_{n}\right)$ and, for all $\mathbf{V}\left(v_{i}\right) \subseteq \mathbf{V} \subset \mathbf{V}\left(v_{i+1}\right)$ [all $\mathbf{V}\left(v_{i}\right) \subseteq \mathbf{V}$ if $i=n$ ], the function $f$ is $\mathbf{V}\left(v_{i}\right)$-continuous if and only if $f$ is $\mathbf{V}$-continuous if and only if $f$ is not $\mathbf{V}\left(v_{i+1}\right)$-continuous. Here we study $\left(\mathbf{V}_{1}, \mathbf{V}_{2}\right)$-continuity for $\mathbf{V}_{1} \neq \mathbf{V}_{2}$. We consider only functions $f:[a, b] \rightarrow[c, d]$ where $a, b, c, d \in \mathbf{V}(\cdot)$, for simplicity.

(I) $\left(\mathbf{V}_{1}, \mathbf{V}_{2}\right)$-continuity for $\mathbf{V}_{1} \subset \mathbf{V}_{2}$.

Definition 4.2 The function $f$ is $\mathbf{V}$-constant if $(\forall x, y \in \operatorname{dom} f)(f(x) \simeq \mathbf{v} f(y))$.

Proposition 4.3 If $\mathbf{V}_{1} \subset \mathbf{V}_{2}$ and $f$ is $\left(\mathbf{V}_{1}, \mathbf{V}_{2}\right)$-continuous, then $f$ is $\mathbf{V}_{2}$-constant.

Proof Fix a natural number $N \in \mathbf{V}_{2}, N \notin \mathbf{V}_{1}$. For $x, y \in[a, b], x<y$, let $\Delta x:=(y-x) / N$ and $x_{i}:=x+i \cdot \Delta x$ for $i \leq N$. Then $\Delta x \simeq \mathbf{v}_{1} 0$, so $x_{i} \simeq \mathbf{v}_{1} x_{i+1}$, and $f\left(x_{i}\right) \simeq \mathbf{v}_{2} f\left(x_{i+1}\right)$ by $\left(\mathbf{V}_{1}, \mathbf{V}_{2}\right)$-continuity. Let $\varepsilon:=\max \left\{\left|f\left(x_{i+1}\right)-f\left(x_{i}\right)\right|: i<N\right\}$; note that $\varepsilon \simeq \mathbf{v}_{2} 0$. We have $|f(y)-f(x)| \leq \Sigma_{i<N}\left|f\left(x_{i+1}\right)-f\left(x_{i}\right)\right| \leq \varepsilon \cdot N \simeq \mathbf{v}_{2} 0$, because $\varepsilon \simeq \mathbf{v}_{2} 0$ and $N \in \mathbf{V}_{2}$. Hence $f(x) \simeq \mathbf{v}_{2} f(y)$.

Corollary 4.4 Iff is $\left(\mathbf{V}_{1}, \mathbf{V}_{2}\right)$-continuous for $\mathbf{V}_{1} \subset \mathbf{V}_{2}$, then $f$ is $\left(\mathbf{V}, \mathbf{V}_{2}\right)$-continuous for all $\mathbf{V}$, and $\mathbf{V}$-continuous for all $\mathbf{V} \subseteq \mathbf{V}_{2}$.

If $f$ is $\mathbf{V}$-constant for all $\mathbf{V}$, then $f$ is constant. Otherwise, there is a coarsest level $\mathbf{V}$ such that $f$ is not $\mathbf{V}$-constant [by Granularity]; we denote it $\mathbf{V}_{f}$. Then $f$ is $\left(\mathbf{V}_{1}, \mathbf{V}_{2}\right)$ continuous for all $\mathbf{V}_{1}$ and all $\mathbf{V}_{2} \subset \mathbf{V}_{f}$, and is not $\left(\mathbf{V}_{1}, \mathbf{V}_{2}\right)$-continuous for any $\mathbf{V}_{1} \subset \mathbf{V}_{2}$ when $\mathbf{V}_{f} \subseteq \mathbf{V}_{2}$. These observations completely describe the behavior of $\left(\mathbf{V}_{1}, \mathbf{V}_{2}\right)$-continuity for the case $\mathbf{V}_{1} \subset \mathbf{V}_{2}$.

(II) $\left(\mathbf{V}_{1}, \mathbf{V}_{2}\right)$-continuity for $\mathbf{V}_{2} \subset \mathbf{V}_{1}$

If $f$ is $\mathbf{V}$-continuous for some $\mathbf{V}$ such that $\mathbf{V}_{2} \subseteq \mathbf{V} \subseteq \mathbf{V}_{1}$, then trivially it is $\left(\mathbf{V}_{1}, \mathbf{V}_{2}\right)$ continuous. Hence it remains to investigate the case when $\mathbf{V}\left(v_{j}\right) \subseteq \mathbf{V}_{2} \subset \mathbf{V}_{1} \subset \mathbf{V}\left(v_{j+1}\right)$ and $f$ is $\mathbf{V}$-discontinuous for all $\mathbf{V}\left(v_{j}\right) \subseteq \mathbf{V} \subset \mathbf{V}\left(v_{j+1}\right)$. 
Let us fix $\mathbf{V}_{2}$ such that $\mathbf{V}\left(v_{j}\right) \subseteq \mathbf{V}_{2} \subset \mathbf{V}\left(v_{j+1}\right)$. Since $f$ is $\mathbf{V}_{2}$-discontinuous,

$$
\left(\exists x, x^{\prime} \in[a, b]\right)\left(x \simeq \mathbf{v}_{2} x^{\prime} \wedge\left|f(x)-f\left(x^{\prime}\right)\right|>\varepsilon\right)
$$

holds for some $\varepsilon>0, \varepsilon \in \mathbf{V}_{2}$.

By Local Transfer, $\left(\exists x, x^{\prime} \in[a, b]\right)\left(x \simeq \mathbf{v} x^{\prime} \wedge\left|f(x)-f\left(x^{\prime}\right)\right|>\varepsilon\right)$ is then true for some $\mathbf{V} \supset \mathbf{V}_{2}$, so $f$ is also $\left(\mathbf{V}, \mathbf{V}_{2}\right)$-discontinuous. This argument also shows that there is no finest level $\mathbf{V}$ such that $f$ is $\left(\mathbf{V}, \mathbf{V}_{2}\right)$-discontinuous.

Each such $\mathbf{V}_{2}$ determines a "cut" in the ordering of levels by $\subseteq$. The "lower class" of the "cut" consists of those $\mathbf{V}$ for which $f$ is $\left(\mathbf{V}, \mathbf{V}_{2}\right)$-discontinuous, and the "upper class" of those for which $f$ is $\left(\mathbf{V}, \mathbf{V}_{2}\right)$-continuous (the latter can be empty). These "cuts" increase with $\mathbf{V}_{2}$ : If $\mathbf{V}_{2} \subseteq \mathbf{V}_{2}^{\prime}$ and $f$ is $\left(\mathbf{V}, \mathbf{V}_{2}\right)$-discontinuous, then $f$ is $\left(\mathbf{V}, \mathbf{V}_{2}^{\prime}\right)$-discontinuous. The "upper class" may or may not have a coarsest "element," as the two following examples show.

Example 1. Let $h \simeq \mathbf{V} 0$ for all $\mathbf{V} \subset \mathbf{V}(h) \supset \mathbf{V}(\cdot)$ [see RST2, Corollary 2.16].

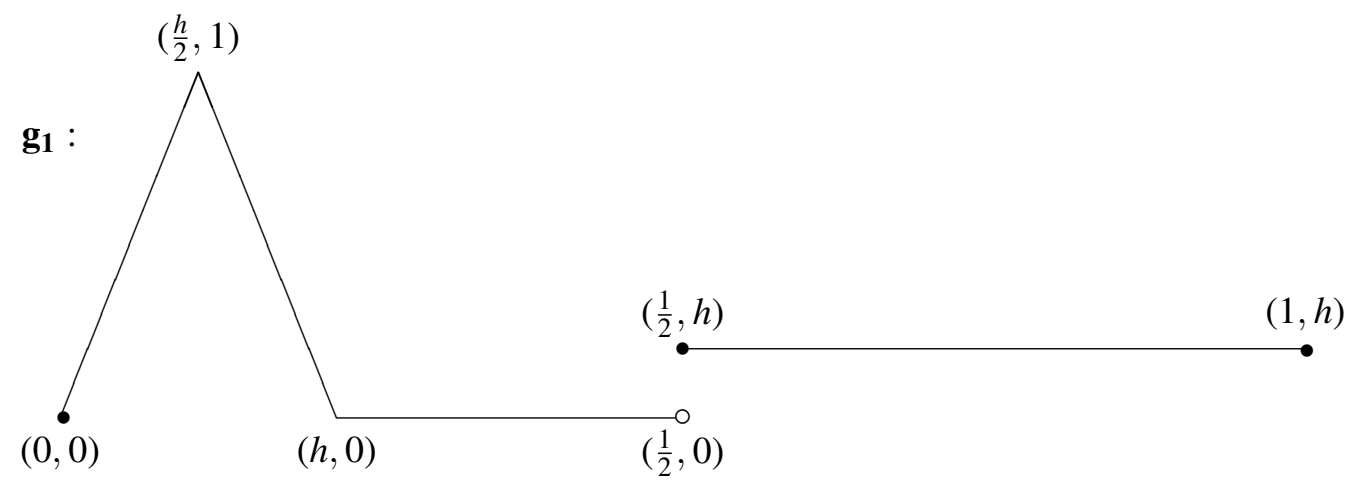

The function $g_{1}$ is $\mathbf{V}$-discontinuous for all $\mathbf{V}$. If $\mathbf{V} \subset \mathbf{V}(h)$, then $g_{1}$ is $(\mathbf{V}, \mathbf{V}(\cdot))$ discontinuous. If $\mathbf{V}(h) \subseteq \mathbf{V}$, then $g_{1}$ is $(\mathbf{V}, \mathbf{V}(\cdot))$-continuous.

Example 2. Let $\left\{v_{0}, \ldots, v_{n}\right\}$ be a level set with $n \notin \mathbf{V}(\cdot)$, and $\left\{h_{0}, \ldots, h_{n}\right\}$ be as in RST2, Corollary 2.16; ie, $\mathbf{V}\left(h_{i}\right)=\mathbf{V}\left(v_{i}\right)$ and $h_{i}$ is ultrasmall relative to all $\mathbf{V} \subset \mathbf{V}\left(h_{i}\right)$. 


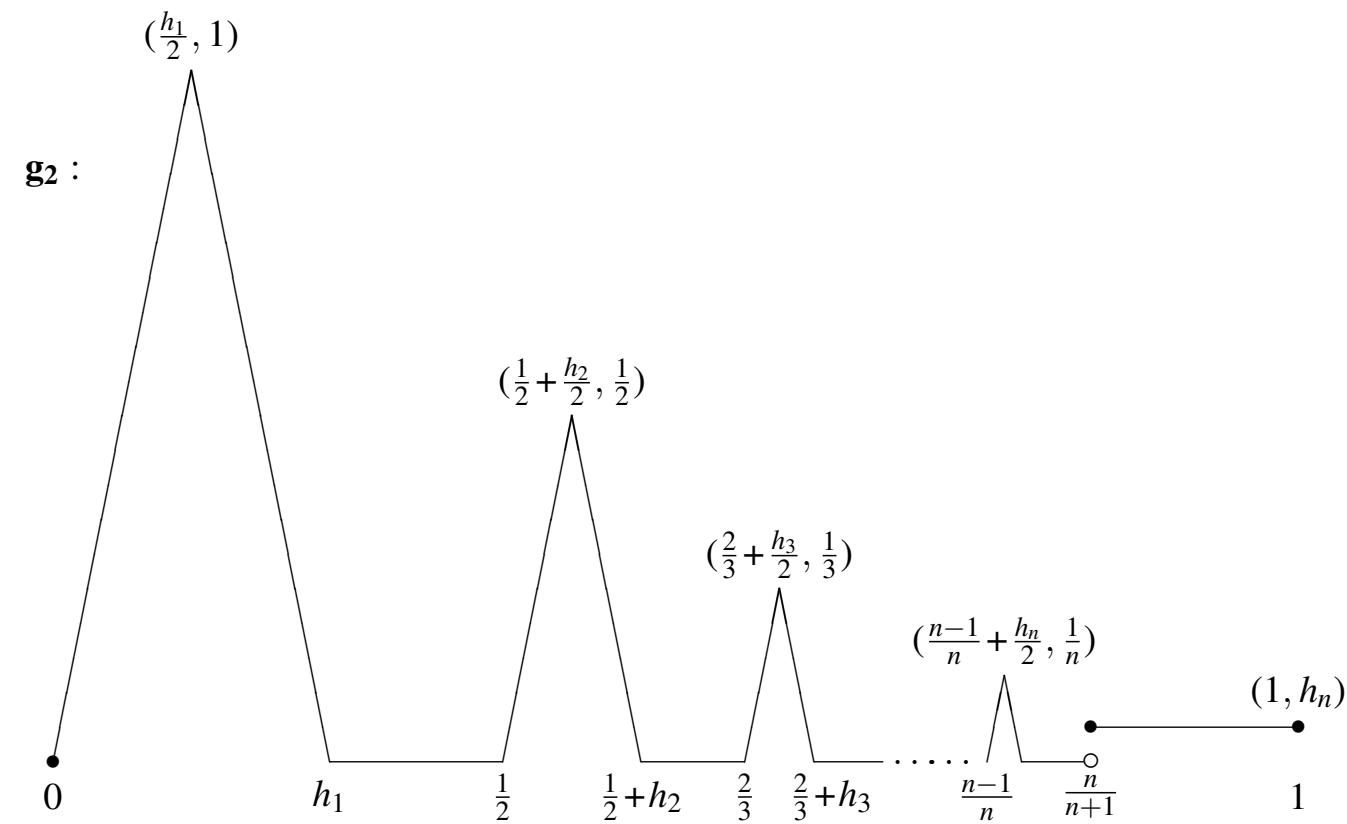

If $\mathbf{V} \subset \mathbf{V}\left(h_{i}\right)$ for some $i \in \mathbf{V}(\cdot)$, then $g_{2}$ is not $(\mathbf{V}, \mathbf{V}(\cdot))$-continuous, because of the spike at $1-1 / i$, of width $h_{i} \simeq \mathbf{V} 0$ and height $1 / i \in \mathbf{V}(\cdot)$.

Otherwise, $\mathbf{V}\left(h_{i}\right) \subseteq \mathbf{V}$ for some $i \notin \mathbf{V}(\cdot)$ [see Proposition 6.2]. As $\left\{h_{0}, \ldots, h_{i-1}\right\} \in$ $\mathbf{V}\left(h_{i-1}\right)$, the function $g_{2}\left\lceil[0,1-1 / i] \in \mathbf{V}\left(h_{i-1}\right)\right.$ [it is definable in $\left.\mathbf{V}\left(h_{i-1}\right)\right]$ and is continuous, ie, $\mathbf{V}\left(h_{i-1}\right)$-continuous, hence $(\mathbf{V}, \mathbf{V}(\cdot))$-continuous. The function $g_{2} \uparrow$ $[1-1 / i, 1]$ is also $(\mathbf{V}, \mathbf{V}(\cdot))$-continuous, because it is bounded by $\max \left\{\frac{1}{i}, h_{n}\right\} \simeq \mathbf{V}(\cdot) 0$. Hence $g_{2}$ is $(\mathbf{V}, \mathbf{V}(\cdot))$-continuous.

Finally, we note that $g_{2}$ is not $\mathbf{V}$-continuous for any $\mathbf{V}$, because of the spike at $1-\frac{1}{i+1}$ if $\mathbf{V}\left(h_{i}\right) \subseteq \mathbf{V} \subset \mathbf{V}\left(h_{i+1}\right)$, and because of the jump at $1-\frac{1}{n+1}$ if $\mathbf{V}\left(h_{n}\right) \subseteq \mathbf{V}$.

\section{Conservativity of variants of relative set theory over BST.}

In GRIST, the ordering of levels by inclusion is dense. In contrast, Discrete GRIST [see RST, Section 12] postulates that the ordering of levels by inclusion is discrete. O'Donovan raised a question of the extent to which these two theories agree. On the one hand, they both are conservative extensions of $\mathbf{Z F C}$, and hence they prove the same $\in$-statements (namely, exactly those that are provable in ZFC). On the other hand, clearly there are statements about levels where the two theories differ. In this section 
we obtain a general result showing that provable statements in which no quantification over levels occurs are exactly the same in GRIST, Discrete GRIST, and other similar theories.

The theory BST is a modification of Nelson's IST [8], introduced by Kanovei (see Kanovei-Reeken [7] and RST, Section 5). The language of BST has $\in$ and a unary predicate $\mathbf{s t}(\cdot)$ (“. is standard”).

If $\mathcal{P}\left(x_{1}, \ldots, x_{n}\right)$ is a formula of the $\in$-st-language, we let $\mathcal{P}\left(x_{1}, \ldots, x_{n} \mid \mathbf{V}\right)$ be the formula obtained from $\mathcal{P}(\bar{x})$ by replacing each occurrence of $\mathbf{s t}(x)$ by $x \in \mathbf{V}$. We note that $\mathcal{P}(\bar{x} \mid \mathbf{V})$ is a $\mathbf{V}$-formula in the sense of RST2, as no quantification over levels occurs in it. Conversely, every formula $\mathcal{Q}(\bar{x}, \mathbf{V})$ of the language of GRIST in which no quantification over levels occurs is of the form $\mathcal{P}(\bar{x} \mid \mathbf{V})$ for some $\in$-st-formula $\mathcal{P}(\bar{x})$. The formula $\mathcal{P}^{\mathrm{W}}(\bar{x} \mid \mathbf{V})$ is obtained from $\mathcal{P}(\bar{x} \mid \mathbf{V})$ by replacing each occurence of $(\forall x)(\ldots)$ by $(\forall x \in \mathbf{W})(\ldots)$ and each $(\exists x)(\ldots)$ by $(\exists x \in \mathbf{W})(\ldots)$.

Let $\mathcal{T}$ be a theory in the language of GRIST. We say that $\mathcal{T}$ is locally BST if

(0) $\mathcal{T} \vdash \mathbf{R}^{\ominus}$ (o - iv) [see RST2], ie, $\mathcal{T}$ proves that levels are linearly ordered by inclusion and there is a coarsest level $\mathbb{S}:=\mathbf{V}(0)=\mathbf{V}(\cdot)$;

(1) $\mathcal{T} \vdash \mathcal{P}(\bar{x} \mid \mathbf{V})$ and $\mathcal{T} \vdash \mathbf{V}_{1} \subset \mathbf{V}_{2} \rightarrow \mathcal{P}^{\mathbf{V}_{2}}\left(\bar{x} \mid \mathbf{V}_{1}\right)$ where $\mathcal{P}(\bar{x})$ is any axiom of BST; and

(2) For every countable model $\mathbf{M}$ of $\mathbf{Z F C}$ there is a countable model $\mathbf{N}$ of $\mathcal{T}$ such that $\mathbf{M}$ is isomorphic to $\mathbf{N} \uparrow \mathbb{S}^{\mathbf{N}}$.

Condition (2) holds if $\mathcal{T}$ is realistic over ZFC in the sense of RST, Section 1. The theories GRIST, Discrete GRIST, and FRIST (more precisely, the theory called FRBST $_{2}$ in [2]) are all locally BST. It is also easy to formulate a "bounded" version of RIST which is locally BST.

Theorem 5.1 If $\mathcal{T}$ is locally BST, then $\mathcal{T} \vdash \mathcal{P}(\bar{x} \mid \mathbf{V})$ if and only if BST $\vdash \mathcal{P}(\bar{x})$.

Proof The "if" direction is an immediate consequence of condition (1) [and the fact that the translation $\mathcal{P}(\bar{x}) \mapsto \mathcal{P}(\bar{x} \mid \mathbf{V})$ preserves logical axioms and deduction rules].

For the "only if" direction, assume that BST $\forall \mathcal{P}(\bar{x})$. Then there is a countable model $\mathbf{P}$ of BST $\wedge(\exists \bar{x}) \neg \mathcal{P}(\bar{x})$. Let $\mathbf{M}:=\mathbf{P}\left\lceil\mathbb{S}^{\mathbf{P}}\right.$, where $\mathbb{S}:=\{x: \mathbf{s t}(x)\}$. Condition (2) implies that there is a countable model $\mathbf{N}$ of $\mathcal{T}$ with $\mathbf{N} \backslash \mathbb{S}^{\mathbf{N}}$ isomorphic to $\mathbf{M}$. By condition (1), $\overline{\mathbf{P}}:=\left(|\mathbf{N}|, \in, \mathbb{S}^{\mathbf{N}}\right)$ is a model of BST. In RST, Corollary 5.9, it is proved that if $\mathbf{P}, \overline{\mathbf{P}}$ are countable models of BST with isomorphic standard universes, then $\mathbf{P}$ and $\overline{\mathbf{P}}$ are isomorphic. Hence $\overline{\mathbf{P}} \vDash(\exists \bar{x}) \neg \mathcal{P}(\bar{x})$ and $\mathbf{N} \vDash(\exists \bar{x}) \neg \mathcal{P}(\bar{x} \mid \mathbb{S})$. This shows $\mathcal{T} \forall \mathcal{P}(\bar{x} \mid \mathbf{V})$. 
Theorem 5.1 can be extended to formulas with any finite list of levels. Let $\mathbf{B S T}_{k}$ be the theory in the language with $\in$ and unary predicates $\mathbf{s t}_{1}, \ldots, \mathbf{s t}_{k}$, postulating

(i) $\mathbf{s t}_{1}(x) \rightarrow \mathbf{s t}_{2}(x) \rightarrow \ldots \rightarrow \mathbf{s t}_{k}(x)$, and

(ii) $\mathcal{P}^{i}(\bar{x})$ for $1 \leq i \leq k$, where $\mathcal{P}(\bar{x})$ is any axiom of BST, and $\mathcal{P}^{i}(\bar{x})$ is obtained from $\mathcal{P}(\bar{x})$ by replacing each occurence of $\mathbf{s t}(x)$ by $\mathbf{s t}_{i}(x)$ and, if $i<k$, each $(\forall x)(\ldots)$ by $(\forall x)\left(\mathbf{s t}_{i+1}(x) \rightarrow \ldots\right)$ and each $(\exists x)(\ldots)$ by $(\exists x)\left(\mathbf{s t}_{i+1}(x) \wedge \ldots\right)$.

We note that $\mathbf{B S T}_{1}$ is BST (with st replaced by $\mathbf{s t}_{1}$ ) and $\mathbf{B S T} \mathbf{T}_{k}$ is "BST iterated $k$ times." It follows immediately by induction that if $\mathbf{P}, \overline{\mathbf{P}}$ are countable models of $\mathbf{B S T}_{k}$ with isomorphic standard universes [ie, $\mathbf{P}\left\lceil\left\{x \in|\mathbf{P}|: \mathbf{P} \vDash \mathbf{s t}_{1}(x)\right\}\right.$ is isomorphic to $\overline{\mathbf{P}}\left\lceil\left\{x \in|\overline{\mathbf{P}}|: \overline{\mathbf{P}} \vDash \mathbf{s t}_{1}(x)\right\}\right.$, then $\mathbf{P}$ and $\overline{\mathbf{P}}$ are isomorphic.

Let $\mathcal{P}\left(\bar{x} \mid \mathbf{V}_{1}, \ldots, \mathbf{V}_{k}\right)$ be the formula obtained from $\mathcal{P}$ by replacing each occurence of $\mathbf{s t}_{i}$ by $x \in \mathbf{V}_{i}$, for all $1 \leq i \leq k$. The argument in the proof of Theorem 5.1, with obvious modifications, proves the following theorem.

Theorem 5.2 If $\mathcal{T}$ is locally BST, then

$\mathcal{T} \vdash \mathbf{V}_{1} \subset \mathbf{V}_{2} \subset \ldots \subset \mathbf{V}_{k} \rightarrow \mathcal{P}\left(\bar{x} \mid \mathbf{V}_{1}, \mathbf{V}_{2}, \ldots, \mathbf{V}_{k}\right)$ if and only if $\mathbf{B S T}_{k} \vdash \mathcal{P}(\bar{x})$

Corollary 5.3 If $\mathcal{T}$ is locally BST, then $\mathcal{T} \vdash \mathcal{P}\left(\bar{x} \mid \mathbf{V}_{1}, \mathbf{V}_{2}, \ldots, \mathbf{V}_{k}\right)$ if and only if GRIST $\vdash \mathcal{P}\left(\bar{x} \mid \mathbf{V}_{1}, \mathbf{V}_{2}, \ldots, \mathbf{V}_{k}\right)$.

Proof For $k=2$ the formula $\mathcal{P}\left(\bar{x} \mid \mathbf{V}_{1}, \mathbf{V}_{2}\right)$ is equivalent to

$$
\left(\mathbf{V}_{1} \subset \mathbf{V}_{2} \rightarrow \mathcal{P}\left(\bar{x} \mid \mathbf{V}_{1}, \mathbf{V}_{2}\right)\right) \wedge\left(\mathbf{V}_{2} \subset \mathbf{V}_{1} \rightarrow \mathcal{P}\left(\bar{x} \mid \mathbf{V}_{1}, \mathbf{V}_{2}\right)\right) \wedge \mathcal{P}\left(\bar{x} \mid \mathbf{V}_{1}, \mathbf{V}_{1}\right)
$$

The claim follows from Theorems 5.2 and 5.1. Similarly for $k>2$.

\section{Corrections and additions to RST.}

The simplified proof of Proposition 6.10 in RST given there does not establish that $T^{\prime} \preccurlyeq \mathcal{U} T$. Below we give the original inductive proof.

Proposition 6.1 (RST, Proposition 6.10) For every $\lambda: \subseteq \Sigma T \rightarrow \omega$ such that $\{t \in \Sigma T: \lambda(t) \leq|t|\} \in \Sigma \mathcal{U}$ there is $T^{\prime} \preccurlyeq \mathcal{U} T$ with $\lambda_{T^{\prime}}=\Sigma \mathcal{U} \lambda$. 
Proof We proceed by induction on the rank of $T$. The claim is clear if $T=\{0\}$.

If $\{t \in \Sigma T: \lambda(t)=0\} \in \Sigma \mathcal{U}$, then $T^{\prime}=\{0\}$ has the required properties. From now on we assume that $\{t \in \Sigma T: \lambda(t)>0\} \in \Sigma \mathcal{U}$.

By RST, Definition 6.2, $X_{0}:=\left\{i:(X)_{\langle i\rangle} \in \Sigma \mathcal{U}_{\langle i\rangle}\right\} \in \mathcal{U}(0)$. For each $i \in X_{0}, s \in(X)_{\langle i\rangle}$ implies $\langle i\rangle \frown s \in X$, so $\lambda(\langle i\rangle \frown s)>0$; let $\lambda_{i}(s)=\lambda(\langle i\rangle \frown s)-1$. By the inductive assumption, for each $i \in X_{0}$ there is $T_{i}^{\prime} \preccurlyeq \mathcal{U}_{\langle i\rangle} T_{\langle i\rangle}$ and a set $X_{i} \subseteq(X)_{\langle i\rangle}, X_{i} \in \Sigma \mathcal{U}_{\langle i\rangle}$, such that $\lambda_{T_{i}^{\prime}}(s)=\lambda_{i}(s)$ for all $s \in X_{i}$.

Now let $T^{\prime}:=\{0\} \cup \bigcup_{i \in X_{0}}\langle i\rangle \frown T_{i}^{\prime}$; clearly $T^{\prime} \preccurlyeq \mathcal{U} T$. The set $Y:=\{\langle i\rangle \frown s: i \in$ $\left.X_{0}, s \in X_{i}\right\} \in \Sigma \mathcal{U}$ and for $t=\langle i\rangle \frown s \in Y, \lambda_{T^{\prime}}(t)=\left|\pi_{T^{\prime}, T}(t)\right|=\left|\pi_{T_{i}^{\prime}, T_{\langle i\rangle}}(t)\right|+1=$ $\lambda_{T_{i}^{\prime}}(s)+1=\lambda_{i}(s)+1=\lambda(t)$.

The next proposition generalizes RST, Proposition 10.5 from pedigrees to level sets.

Proposition 6.2 Let $L=\left\{\gamma_{0}, \ldots, \gamma_{n}\right\}$ be a level set. For every $x$ either $x \sqsubset \gamma_{0}$ or $\gamma_{i} \sqsubseteq x \sqsubset \gamma_{i+1}$ for some $i<n$, or $\gamma_{n} \sqsubseteq x$.

Proof Either $x \boxminus \gamma_{i}$ for some $i \leq n$, or $L \cup\{x\}$ is a level set, hence well-ordered by $\sqsubseteq$. From this, the claim follows.

The following proposition is often useful for specifying subsets of level sets.

Definition 6.3 A formula $\mathcal{P}(z, \bar{x})$ is stable in $z$ if $\alpha \sqsubset z \rightarrow\left(\mathcal{P}(z, \bar{x}) \leftrightarrow \mathcal{P}^{\alpha}(z, \bar{x})\right)$.

Examples. (1) $\mathcal{P}(z, X): z \sqsubset x$ is stable in $z$. [For $\alpha \sqsubset z, z \sqsubset x \leftrightarrow z \sqsubset \alpha x$.]

(2) Similarly, $\mathcal{P}(z, X):(\exists x \in X)(x \boxminus z)$ is stable in $z$.

(3) $\mathcal{P}(z, X):(\forall v)[z \sqsubset v \rightarrow(\exists x \in X)(z \sqsubset x \sqsubseteq v)]$ is also stable in $z$.

Proposition 6.4 Let $\mathcal{P}(z, \bar{x})$ be stable in $z$. For every $\bar{x}$ and every level set $L$ there is a (level) set $M$ such that $(\forall z)(z \in M \leftrightarrow z \in L \wedge \mathcal{P}(z, \bar{x}))$.

Proof Let $L=\left\{\gamma_{0}, \ldots, \gamma_{n}\right\}$; we consider the statement

$$
\mathcal{Q}^{\alpha}(L, \bar{x}): \quad(\exists N)(\forall z)\left(z \in N \leftrightarrow z \in L \wedge z \sqsupset_{\alpha} 0 \wedge \mathcal{P}^{\alpha}(z, \bar{x})\right)
$$

and use Granularity to prove $\mathcal{Q}^{0}(L, \bar{x})$. 
The statement is true when $L \sqsubseteq \alpha$, with $N=\varnothing$. [Recall that $L \sqsubseteq \alpha$ implies $z \sqsubseteq \alpha$ for all $z \in L$; see RST2, Proposition 1.10 (13); also note that $L \boxminus \gamma_{n}$.]

By Granularity, there is a $\sqsubseteq$-least level $\alpha$ for which $\mathcal{Q}^{\alpha}(L, \bar{x})$ holds; by the above, $\alpha \sqsubseteq \gamma_{n} \boxminus L$.

By Proposition 6.2, either $\alpha \sqsubset \gamma_{0}$ or $\gamma_{i} \sqsubseteq \alpha \sqsubset \gamma_{i+1}$ for some $i<n$, or $\alpha \boxminus \gamma_{n}$.

In the first case, $\mathcal{Q}^{\alpha}(L, \bar{x})$ clearly implies $\mathcal{Q}^{0}(L, \bar{x})$, with the same $N$. In the second case, $\mathcal{Q}^{\alpha}(L, \bar{x})$ implies $\mathcal{Q}^{\gamma_{i}}(L, \bar{x})$ (with the same $N$ ), so $\alpha \boxminus \gamma_{i}$. Let $\gamma_{i-1} \sqsubseteq \beta \sqsubset \gamma_{i}$ [ $\beta \sqsubset \gamma_{i}$ if $i=0$ and $\gamma_{i} \sqsupset 0$ ]. By stability of $\mathcal{P}$ in $z, \mathcal{P}^{\beta}(z, \bar{x}) \leftrightarrow \mathcal{P}(z, \bar{x}) \leftrightarrow \mathcal{P}^{\alpha}(z, \bar{x})$ for all $z \sqsupset \alpha$. If $\mathcal{P}^{\beta}\left(\gamma_{i}, \bar{x}\right)$, let $N^{\prime}:=N \cup\left\{\gamma_{i}\right\}$; otherwise let $N^{\prime}:=N$. Then $\mathcal{Q}^{\beta}(L, \bar{x})$ holds (with $N^{\prime}$ in place of $N$ ), and we have a contradiction. Thus $\alpha \boxminus \gamma_{0} \boxminus 0$, and $\mathcal{Q}^{0}(L, \bar{x})$ holds in this case, too. The third case is like the second, with $i=n$.

Let now $N$ be such that $(\forall z)(z \in N \leftrightarrow z \in L \wedge z \sqsupset 0 \wedge \mathcal{P}(z, \bar{x}))$.

We set $M:=N \cup\left\{\gamma_{0}\right\}$ if $\gamma_{0} \boxminus 0 \wedge \mathcal{P}\left(\gamma_{0}, \bar{x}\right)$, and $M:=N$ otherwise. Clearly $M$ has the required properties.

In RST, Corollary 12.7, the completeness of GRIST over ZFC is formulated as follows:

If $\mathbf{T} \supseteq \mathbf{Z F C}$ is a complete consistent theory (in the $\in$-language), then $\mathbf{T}+$ GRIST is a complete consistent theory (in the $\in-\sqsubseteq$-language).

Here we give a reformulation that is perhaps more striking.

Theorem 6.5 Let $\mathcal{P}$ be any formula (in the $\epsilon-\sqsubseteq$-language). If GRIST $+\mathcal{P}$ is a conservative extension of ZFC, then GRIST $\vdash \mathcal{P}$.

Proof Assume that GRIST $+(\neg \mathcal{P})$ is consistent. Let $\overline{\mathbf{G}}$ be a complete consistent extension of this theory, and let $\overline{\mathbf{T}}$ be the restriction of $\overline{\mathbf{G}}$ to formulas in the $\in$-language. Then $\overline{\mathbf{T}} \supset \mathbf{Z F C}$ is complete and consistent. By RST, Corollary 12.7, $\overline{\mathbf{T}}+$ GRIST is complete and consistent, so $\overline{\mathbf{T}}+$ GRIST $=\overline{\mathbf{G}}$ and $\overline{\mathbf{T}}+$ GRIST $\vdash \neg \mathcal{P}$. Hence $\overline{\mathbf{T}}+$ GRIST $+\mathcal{P}$ is inconsistent. It follows that GRIST $+\mathcal{P} \vdash \neg \mathcal{Q}$ for some $\mathcal{Q} \in \overline{\mathbf{T}}$. Since GRIST $+\mathcal{P}$ is assumed to be a conservative extension of $\mathbf{Z F C}$, we have also $\mathbf{Z F C} \vdash \neg \mathcal{Q}$ and $\neg \mathcal{Q} \in \overline{\mathbf{T}}$, a contradiction. 


\section{References}

[1] P Andreev, private communication.

[2] K Hrbacek, Internally iterated ultrapowers, in Nonstandard Models of Arithmetic and Set Theory, ed. by A Enayat and R Kossak, Contemporary Math. 361, American Mathematical Society, Providence, RI, 2004, 87-120.

[3] K Hrbacek, Stratified analysis?, in The Strength of Nonstandard Analysis, ed. by I van den Berg and V Neves, Springer Wien New York, 2007, 47-63.

[4] K Hrbacek, Relative set theory: Internal view, Journal of Logic and Analysis 1:8 (2009), 1-108. doi: 10.4115/jla.2009.1.8.

[5] K Hrbacek, Relative set theory: Some external issues, Journal of Logic and Analysis 2:8 (2010), 1-37. doi: 10.4115/jla.2010.2.8.

[6] K Hrbacek, O Lessmann and R O'Donovan, Analysis with ultrasmall numbers, Amer. Math. Monthly 117 (2010), 801-816. doi: 10.4169/000298910X521661.

[7] V Kanovei and M Reeken, Nonstandard Analysis: Axiomatically, Springer-Verlag Berlin Heidelberg New York, 2004, xvi +408 pages.

[8] E Nelson, Internal set theory: a new approach to Nonstandard Analysis, Bull. Amer. Math. Soc. 83 (1977), 1165-1198; doi: 10.1090/S0002-9904-1977-14398-X.

[9] Y Péraire, Théorie relative des ensembles internes, Osaka J. Math. 29 (1992), 267-297.

[10] Y Péraire, Infinitesimal approach of almost-automorphic functions, Ann. Pure Appl. Logic 63 (1993), 283-297; doi: 10.1016/0168-0072(93)90152-4.

The City College of New York

khrbacek@sci.ccny. cuny. edu

Received: 25 April 2011 Revised: 16 May 2012 